\title{
Consideraciones sobre los efectos de incorporar costos de transacción fijos y proporcionales en la valoración de opciones financieras por el modelo Cox, Ross, Rubinstein
}

About effects of incorporating fixed and proportional transaction costs in the valuation of financial options by the Cox, Ross, Rubinstein model

Sergio Andrés Mendoza Jaimes*

* Magíster en finanzas. Analista de planeación financiera, Ministerio de Ambiente y Desarrollo Sostenible, Bogotá (Colombia). [sergiomendoza.jaimes@gmail.com].

Artículo recibido el 15 de octubre de 2018.

Aceptado el 15 de noviembre de 2018.

Para citar este artículo:

Mendoza Jaimes, S. A. (2018). Consideraciones sobre los efectos de incorporar costos de transacción fijos y proporcionales en la valoración de opciones financieras por el modelo Cox, Ross, Rubinstein. ODEON, 15, 7-52.

DOI: https://doi.org/10.18601/17941113.n15.02 



\section{Introducción}

El modelo presentado por Cox, Ross y Rubinstein (CRR) (1979), conocido como binomial, muestra una aproximación en tiempo discreto finito al modelo de Black-Scholes en la valoración de opciones financieras, el cual mejora su ajuste cuando hay tendencias definidas en el activo subyacente y un mayor número de periodos de revisión (Berk y Demarzo, 2008).

El modelo binomial se ajusta a los supuestos del mercado perfecto que lo asume libre de fricciones; para efectos de este documento, sin costos de transacción. Esto garantiza que las comisiones a intermediarios no impacten en el valor de la prima. Si bien teóricamente esto puede ser válido, en la toma práctica de decisiones es un supuesto bastante fuerte, ya que en los mercados es necesario contemplar el impacto de los costos de transacción, ya sean proporcionales al monto de la transacción o fijos; dicho impacto se puede intensificar conforme aumenta el número de periodos de revisión.

Desde una perspectiva metodológica, se resalta que el modelo CRR para la valoración de opciones financieras sigue un proceso denominado inducción hacia atrás (backward induction) desde el momento en que se liquida la posición o el tiempo de vencimiento, hasta el momento inicial donde se negocia el instrumento, realizando en cada uno de los nodos una cobertura dinámica o ajuste de posiciones, que permite ir construyendo un portafolio de réplica que representa en el instante inicial la prima de la opción.

Siguiendo este orden de ideas, el objetivo de este documento es, a partir del desarrollo del modelo CRR con costos de transacción, analizar el efecto que tiene la inclusión de estos en el valor de las opciones financieras. Para ello se simularán y posteriormente se contrastarán los modelos con y sin costos de transacción; dichos costos surgen de la intermediación de un creador de mercado (market maker), que se asumen separables y simétricos, y que se modelarán a través del spread bid/ask.

Por último, y con el fin de tener un mayor alcance en el análisis y un mayor sustento en las conclusiones, el modelo se evaluará para diferentes escenarios, entre ellos la valoración de opciones europeas y americanas tanto call como put.

\section{El modelo Cox, Ross, Rubinstein}

El modelo CRR para la valoración de opciones financieras realiza un proceso de inducción hacia atrás (backward induction) en el cual construye un portafolio 
de replicación del valor de la opción en cada periodo desde el tiempo de vencimiento o liquidación de la posición hasta el tiempo inicial. Es precisamente durante este proceso donde se incluirán los costos de transacción para cada uno de los periodos definidos.

Entendiendo los periodos como el conjunto finito de tiempos o fechas de negociación donde se ramificará el árbol binomial de acuerdo con los dos posibles estados de la naturaleza probables que describen el comportamiento del precio del activo subyacente, a continuación se analizará el modelo CRR para uno $T=\{0,1\}$ o varios periodos $T=\{0, \mathrm{~N}\}$.

\subsection{Modelo Cox, Ross, Rubinstein de un periodo}

Para iniciar la descripción del modelo CRR de un periodo, se asume la existencia de los siguientes activos:

Activo libre de riesgo: se concibe un préstamo o un bono cuyo precio se denotará $B_{t}$. Este dependerá del tiempo y de la tasa libre de riesgo de la siguiente forma: $B_{t}=B_{t-1}\left(1+r_{f}\right)$; donde $r_{f}$ es la tasa libre de riesgo, la cual se asume como conocida y constante en el tiempo. Para efectos del desarrollo de este documento se asumirá el valor del activo libre de riesgo en el instante inicial como $B_{0}=1$, de esta forma, $B_{0}=\left(1+r_{f}\right)$.

Activo subyacente o riesgoso: para efectos de este documento se concibe como una acción, el cual se denotará $S_{t}$ y se comporta bajo un proceso binomial multiplicativo. Es decir que, en el siguiente instante solo podrá tomar dos posibles valores y estará definido por $S_{t}=X_{i} S_{0}$ aquí $X_{i}$ es una variable que describe su tasa de crecimiento $(u)$ o decrecimiento $(d)$. Donde:

$$
u>1+\mathrm{r}_{f}>d>0
$$

La desigualdad (1) garantiza que no existan oportunidades de arbitraje en el modelo CRR. Para entender esta condición se realiza el siguiente análisis: si $u>d>1+r_{f}$ se dice que hay oportunidad de arbitraje, ya que se podría tomar un préstamo por valor $S_{0}$ a la tasa libre de riesgo para comprar el activo subyacente en $t=0$, activo que en el instante $t=T$ valdrá $S_{T}$, lo que generará en cualquiera de los casos el rendimiento suficiente para pagar el préstamo y obtener ganancia. De la misma forma, si $1+r_{f}>u>d$ se podría arbitrar vendiendo el activo subyacente en el instante 
inicial e invirtiendo este valor a la tasa libre de riesgo, ya que en un instante $t=T$ se tendrá un rendimiento suficiente para cubrir la posición y obtener ganancia. Finalmente, el hecho de que $\mathrm{d}>0$ implica que siempre se van a representar precios positivos.

Asimismo, cabe destacar que la posibilidad de que se materialice un estado de la naturaleza u otro está definida por las probabilidades $q_{u}$ y $q_{d}$, las cuales serán relevantes en la construcción del árbol binomial que representa el precio del activo subyacente de la siguiente forma.

$$
S_{1}\left\{\begin{array}{l}
u S_{0}, \text { con Probabilidad } q_{u} \\
d S_{0}, \text { con Probabilidad } q_{d} \text { o }\left(1-q_{u}\right)
\end{array}\right.
$$

\subsection{Supuestos generales}

Los siguientes son los supuestos generales presentados por el modelo CRR:

- El mercado es libre de fricciones: no existen costos de transacción, tasas impositivas o comisiones. Será precisamente en este supuesto en el cual se centrará este documento, agregando en el capítulo 3 la fricción que conllevan los costos de transacción.

- Los activos son perfectamente divisibles: se puede negociar cualquier cantidad de activos.

- Tasa libre de riesgo $r_{f}$ : es la misma para invertir o tomar prestado, es constante en el tiempo y es conocida.

- Ventas en corto: se pueden realizar ventas en corto, es decir, se pueden contraer obligaciones hoy sobre un activo que no se posee con el compromiso de cumplir la obligación en el futuro.

\subsection{Valoración por replicación}

Esta metodología soporta el modelo CRR, y consiste en construir durante el proceso de inducción hacia atrás el portafolio que replique el valor de la opción a partir del rebalanceo de posiciones. Para esto, el modelo debe establecer la condición de no arbitraje (1) y así garantizar el cumplimiento de la Ley de único precio. En este orden de ideas, lo primero es entender los siguientes términos: 


\subsubsection{Portafolio}

El portafolio $V_{t}\left(\delta_{i}\right)$ se entiende como una cartera conformada por dos activos $\delta_{i}=y, x$, donde $y$ representa el monto invertido a la tasa libre de riesgo, y $x$ el número de acciones. De esta manera, el valor de un portafolio en el tiempo $t$ estará dado por:

$$
V_{t}=y B_{t}+x S_{t}
$$

Teniendo en cuenta la conformación del portafolio es posible asumir que su valor en el tiempo inicial $V_{0}$ es conocido, ya que $S_{0}$ es igual al Spot y se asumió $B_{0}=1$.

\subsubsection{Rebalanceo de posiciones}

El rebalanceo de posiciones permite al inversionista gestionar el riesgo durante la negociación de opciones financieras, ya que le suministra información relevante para realizar el ajuste en la posición de activo riesgoso a través del cálculo del delta $(\Delta)$ y la velocidad con la cual debe realizarlos por medio de Gamma $(\Gamma)$, ambos efectos ante cambios en el precio del activo subyacente.

A continuación, se presenta la fórmula de cálculo de delta $(\Delta)$ en tiempo discreto para un modelo binomial de un periodo (Wallner y Wystup, 2004, pp. 6-7):

$$
\Delta \approx \frac{V_{T=1}^{u}-V_{T=1}^{d}}{S_{0}(u-d)}
$$

Así, $V_{T=1}^{u}$ y $V_{T=1}^{d}$ son los valores del portafolio de réplica, y $S_{0}(u-d)$ los precios del activo subyacente en el momento $T=1$, según los dos posibles estados de la naturaleza.

Por su parte, la fórmula de cálculo de Gamma $(\Gamma)$ en tiempo discreto siguiendo el modelo binomial de dos periodos es (Wallner y Wystup, 2004, pp. 6-7):

$$
\Gamma \approx \frac{\frac{V_{T=2}^{u^{2}}-V_{T=2}^{u d}}{S_{0}\left(u^{2}-1\right)}-\frac{V_{T=2}^{u d}-V_{T=2}^{d^{2}}}{S_{0}\left(1-d^{2}\right)}}{S_{0}\left(u^{2}-d^{2}\right)}
$$




\subsubsection{No arbitraje}

Se considera que un portafolio $V_{t}$ genera posibilidad de arbitraje cuando permite obtener ganancias futuras sin una inversión inicial y sin asumir ningún riesgo ${ }^{1}$.

Ley de único precio: considerando dos activos A y B con precios $S_{0}^{A} \geq 0$ y $S_{0}^{B} \geq 0$ en $t=0$, negociados en un mercado libre de oportunidades de arbitraje. Si en algún $T \geq 0$, en todos los estados de la naturaleza, entonces $S_{T}^{A}=S_{T}^{B}$ (Moreno, 2015, pp. 5-15).

Para entender mejor el concepto se analizará el modelo de un periodo, valorando el portafolio en los instantes, $t=0$ y $T=1$. De esta manera, tenemos en $t=0$ :

$$
V_{0}=y+x S_{0}=Z_{t=0}
$$

Por su parte, en un momento $T=1$ puede tomar los siguientes valores:

$$
\begin{gathered}
V_{T=1}^{u}=x u S_{0}+y\left(1+r_{f}\right) \\
V_{T=1}^{d}=x d S_{0}+y\left(1+r_{f}\right)
\end{gathered}
$$

Dada la ley de único precio, en $T=1$ se tiene que:

$$
\left\{\begin{array}{l}
V_{T=1}^{u}=x u S_{0}+y\left(1+r_{f}\right)=Z_{T=1}^{u}=f\left(u S_{0}\right) \\
\underbrace{V_{T=1}^{d}=x d S_{0}+y\left(1+r_{f}\right)}_{\text {PORTAFOLIO DE REPÚBLICA }}=\underbrace{Z_{T=1}^{u}}_{\text {DERIVADO }}=f\left(u S_{0}\right)
\end{array}\right.
$$

Donde: $Z_{1}^{X_{i}}$ es el derivado. De esta manera, podemos decir que el valor del derivado puede expresarse principalmente como una función del precio del activo subyacente al momento de ejercicio de la opción: $f\left(X_{i} S_{0}\right)$, con $X_{i}=u, d$ para un periodo. Donde la forma de la función $(f)$ determina el tipo de derivado que se está considerando (Moreno, 2015, pp. 5-15).

Dado que el sistema de ecuaciones tiene solución única debido a que cuenta con igual número de ecuaciones y variables, además de que estas no son

1 Existe la posibilidad de arbitraje si: $V_{0}=0, P\left(V_{r} \geq 0\right)=1$ y $P\left(V_{r}>0\right)>0$. 
linealmente dependientes por la condición de no arbitraje, es posible despejarlas para hallar el número de acciones $x$ y el monto invertido a la tasa libre de riesgo $y$, para llegar así a:

$$
x=\frac{f\left(u S_{0}\right)-f\left(d S_{0}\right)}{(u-d) S_{0}}
$$

De igual forma se puede concluir que:

$$
y=\left(\frac{1}{1+r_{f}}\right) \frac{u f\left(d S_{0}\right)-d f\left(u S_{0}\right)}{(u-d)}
$$

Dadas las cantidades halladas, reemplazándolas en la ecuación (6) del portafolio, se obtiene que:

$$
V_{0}=Z_{0}=\frac{1}{1+r_{f}}\lceil\underbrace{\left(\frac{1+r_{f}-d}{u-d}\right)}_{q_{u}} f\left(u S_{0}\right)+\underbrace{\left(\frac{u-1-r_{f}}{u-d}\right)}_{q_{d}} f\left(d S_{0}\right)\rceil
$$

Las expresiones $q_{u}$ y $q_{d}$ se pueden entender como probabilidades debido a que son cantidades positivas dada la condición de no arbitraje, y su suma es 1 . Lo anterior permite sintetizar el valor de la opción en el siguiente valor esperado:

$$
V_{0}=Z_{0}=\frac{1}{1+r_{f}}\left\lceil q_{u} f\left(u S_{0}\right)+q_{d} f\left(d S_{0}\right)\right\rceil
$$

Se denominan probabilidades de riesgo neutral debido a que bajo esta medida el valor esperado de la rentabilidad de todos los activos es igual a la tasa libre de riesgo, como se muestra a continuación para el caso del activo riesgoso (Moreno, 2015, pp. 5-15):

$$
E^{Q}\left[S_{1}\right\rceil=\left(\frac{1+r_{f}-d}{u-d}\right) u S_{0}+\left(\frac{u-1-r_{f}}{u-d}\right) d S_{0}=S_{0}\left(1+r_{f}\right)
$$




\subsubsection{Modelo de valoración generalizado}

Una vez descrito el modelo CRR para un periodo, se realiza la generalización al modelo de valoración para cualquier número de periodos de revisión ( $n$ ) que se definan durante la vida del contrato. De esta manera, al describir por separado el comportamiento del activo libre de riesgo y subyacente, se tiene que:

\section{Activo libre de riesgo:}

$$
B_{1}=B_{0}\left(1+r_{f}\right) ; B_{2}=B_{0}\left(1+r_{f}\right)^{2} ; B_{n}=B_{0}\left(1+r_{f}\right)^{n}
$$

Activo subyacente para $\boldsymbol{n}>1$ : para extender el modelo a más periodos, se asume que la volatilidad del activo subyacente será constante durante la vigencia del contrato. De esta manera, también lo serán la tasa de crecimiento $u \mathrm{y}$ decrecimiento $d$, y, consecuentemente, las probabilidades $q_{u}$ y $q_{d}$. Dado esto, es pertinente decir que, para cada ramificación del árbol, el modelo se comporta como un modelo CRR de un paso.

Realizando el análisis para $n=2$ tenemos que:

En un escenario alcista en un $t=1$

$$
Z_{1}^{u}=\frac{1}{1+r_{f}}\left\lceil q_{u} Z_{2}^{u u}+q_{d} Z_{2}^{u d}\right\rceil
$$

En el escenario contrario tenemos:

$$
Z_{1}^{d}=\frac{1}{1+r_{f}}\left\lceil q_{u} Z_{2}^{u d}+q_{d} Z_{2}^{d d}\right\rceil
$$

Utilizando una vez más la fórmula de valoración se tiene que el valor del derivado en $t=0$ es:

$$
Z_{0}=\frac{1}{1+r_{f}}\left\lceil q_{u} Z_{1}^{u}+q_{d} Z_{1}^{d}\right\rceil
$$


Teniendo en cuenta las ecuaciones anteriores, obtenemos:

$$
Z_{0}=\frac{1}{1+r_{f}}\left\lceil q_{u} \frac{1}{1+r_{f}}\left\lceil q_{u} Z_{2}^{u u}+q_{d} Z_{2}^{u d}\right\rceil+q_{d} \frac{1}{1+r_{f}}\left\lceil q_{u} Z_{2}^{u d}+q_{d} Z_{2}^{d d}\right\rceil\right\rceil
$$

Lo cual se puede reducir a:

$$
Z_{0}=\frac{1}{\left(1+r_{f}\right)^{2}} \sum_{k=0}^{2}\left(\begin{array}{l}
2 \\
k
\end{array}\right) q_{u}{ }^{2-k} q_{d}{ }^{k} Z_{1}^{u^{2-k} d^{k}}
$$

De esta manera, se podría generalizar el modelo como:

$$
Z_{0}=\frac{1}{\left(1+r_{f}\right)^{N}} \sum_{k=0}^{N}\left(\begin{array}{c}
N \\
k
\end{array}\right) q_{u}{ }^{N-k} q_{d}{ }^{k} Z_{N}^{u^{N-k} d^{k}}
$$

Donde: $Z_{N}^{u^{N-k} d^{k}}=f\left(u^{N-k} d^{k} S_{0}\right)$

El valor del derivado en el modelo generalizado estará en función del precio del activo subyacente al vencimiento, siendo $u^{N-k} d^{k}$ la expresión que describe el comportamiento del precio durante la vigencia del contrato $(0 \leq t \leq T)$. De esta manera podemos afirmar que el modelo generalizado es un proceso reiterativo del modelo de un periodo, el cual con su llegada al instante inicial replicará el valor de la prima de las opciones financieras.

\section{El modelo Cox, Ross, Rubinstein con costos de transacción}

Para realizar la valoración de opciones financieras, el modelo CRR hace un ajuste de posiciones en cada nodo. Sin embargo, en la práctica los inversionistas no suelen hacer ajuste de posiciones con gran periodicidad, debido a la inexistencia de una contraparte o a la presencia de costos de transacción.

En este capítulo se obtendrán las ecuaciones que Tichý (2005) denominó universales, a partir de las cuales se podrán derivar las ecuaciones que permitan construir el portafolio de réplica de las opciones call y put en sus posiciones 
largas y cortas. Expresiones que además de considerar el ajuste de posiciones en cada nodo garantizan la cobertura de los costos derivados del mismo.

\subsection{Inexistencia de contraparte}

La inexistencia de contraparte se entiende como la dificultad que puede tener el inversionista de encontrar una contraparte que esté dispuesta a efectuar las transacciones contrarias en cortos periodos de tiempo. Se puede expresar como una restricción a la liquidez del activo subyacente en el ajuste de posiciones en el cual se soporta el modelo CRR, ya que, si bien en los mercados financieros convergen gran cantidad de actores, no existe seguridad de que pueda tomar ni las posiciones ni las cantidades que le demanda el rebalanceo.

Una vez entendida la restricción, es importante resaltar que esta no será objeto de estudio en el presente documento, ya que se asumirá que se actuará en un mercado dirigido por precios en el cual el intermediario financiero, en este caso el creador de mercado (market maker), garantizará la existencia de contraparte en el mercado en cada uno de los periodos de revisión, ya sea buscándola con otros inversionistas o asumiéndola él.

\subsection{Presencia de costos de transacción}

Al incluir en el modelo CRR los costos de transacción, intuitivamente podemos inferir que el valor de la opción se verá afectado, lo cual hace que la posición sea menos rentable para cada posición, debido a las fricciones del mercado. Para esto se consideró representar los costos de transacción a través del diferencial de precios (spread bid/ask) del activo subyacente en el mismo instante de tiempo, siendo ask el de compra y bid el de venta. Dicho diferencial de precios es generado por la intermediación que realiza el creador de mercado, y representa, ya sean los costos operativos, costos de cartera o costos de selección adversa (Stoll, 1989), es decir, una prima por el riesgo asumido en cada una de las negociaciones.

Para la implementación se asumirán costos de transacción separables, es decir, la presencia de un tipo de costos no necesariamente implicaría que se causen otros. Además, se asumirán simétricos tanto los costos fijos como los proporcionales al monto de la transacción, es decir, la diferencia en valor absoluto entre el precio de venta y el precio de mercado será igual a la diferencia entre el precio de mercado y el de compra. Y serán representados como: $S^{a s k}$ y $S^{\text {bid }}$, 
donde:

$$
S_{t}(1-k)=S^{a s k}<S_{t}<S^{b i d}=S_{t}(1+k)
$$

Es decir que $S^{b i d}$ es estrictamente más alto que $S^{a s k}$ en un mismo instante de tiempo (Tichý, 2005), y donde $k$ es el porcentaje de costos de transacción, ya sean costos fijos $k_{f}$ o proporcionales $k_{p}$.

Finalmente, asumiendo el portafolio replicante como autofinanciado, es decir, en ningún momento de la vida del contrato se inyectarán nuevos recursos por parte del inversionista, será posible conocer el valor de la opción en cada uno de los nodos a partir de su valor en la fecha de vencimiento. Y será en la inducción hacia atrás donde se irán incorporando nodo a nodo los costos de transacción como una provisión para cubrir los costos del rebalanceo. Además de conocer el valor del portafolio será necesario conocer cuánto es invertido en el activo riesgoso y cuánto en el libre de riesgo (Boyle y Vorst, 1992).

\subsubsection{Modelo con costos de transacción proporcionales al monto comercializado $\left(k_{p}\right)$}

Para incluir estos costos de transacción es necesario entender que los mismos son causados cuando el activo riesgoso es comercializado. Teniendo en cuenta que los costos de transacción son proporcionales $k_{p}$, estos serán calculados como un porcentaje del monto comercializado. Para efectos del presente documento se asume que el inversionista cuenta con la posición inicial en el activo riesgoso $x_{0}$, es decir, no habrá necesidad de hacer un primer ajuste de posiciones en el portafolio de replicación. Por lo tanto, solo se tienen en cuenta los costos adicionales requeridos para mantener el portafolio de replicación.

$$
\begin{gathered}
t=0 V_{0}\left(x_{0}, y_{0}\right)=x_{0} S_{0}+y_{0} B \\
x_{T-1} S_{T-1}+B_{0}\left(1+r_{f}\right)^{T-1}=Z_{T}^{u}+k_{P}\left|X_{T-1}-X_{T}^{u}\right| u S_{T-1} \\
x_{T-1} S_{T-1}+B_{0}\left(1+r_{f}\right)^{T-1}=Z_{T}^{d}+k_{P}\left|X_{T-1}-X_{T}^{d}\right| d S_{T-1}
\end{gathered}
$$

La primera expresión de las ecuaciones no lineales (23) (24), que se encuentra a la izquierda, representa el valor del portafolio que replica la opción en $t=T-1$, mientras la segunda expresión hace referencia a la suma entre el valor 
del portafolio en el siguiente periodo $(T)$, el cual, al tratarse del vencimiento de la opción europea, será equivalente al $\operatorname{payoff}\left(Z_{T}^{X_{i}}\right)$, y la provisión necesaria para cubrir los costos proporcionales derivados del rebalanceo $\left(k_{P}\left|x_{T-1}-x_{T}^{X_{i}}\right| X_{i} S_{T-1}\right)$, donde:

$k_{p}$ : porcentaje de costos proporcionales al monto de la transacción, el cual fija el creador de mercado.

$X_{i} S_{T-1}$ : precio del activo subyacente en el periodo $T$, el cual depende del estado de la naturaleza $X_{i}$ que se materialice, ya sea alcista $(u)$ o bajista $(d)$.

$\left|x_{T-1}-x_{T}^{X_{i}}\right|$ : diferencia en número de acciones entre los dos periodos, la cual se expresa en valor absoluto para representar tanto un escenario alcista como uno bajista, lo cual tiene repercusiones diferentes de acuerdo con el tipo de la opción y su posición.

De esta manera, el valor del portafolio en cualquiera de los dos estados que se presenten será suficiente para realizar el ajuste de posiciones que demanda el portafolio y cubrir los costos que se derivan de esta transacción.

Finalmente, tomando como ejemplo la desigualdad presentada en el teorema 1 del documento de Phelim Boyle y Ton Vorst (1992), que ofrece una única solución para las ecuaciones no lineales (23) y (24), durante la construcción del portafolio de réplica de una opción europea larga de compra (long european call option) por cobertura dinámica, en el presente documento se desarrolló la extensión de las desigualdades para las posiciones largas y cortas de opciones de venta (long and short european put options) y compra (long and short european call options), las cuales se presentan en la tabla 1.

Tabla 1: Desigualdades que garantizan solución única de opciones europeas de venta y compra

\begin{tabular}{|c|c|c|}
\hline Posición-Opción & Call & Put \\
\hline Larga & $x_{T}^{d} \leq x_{T-1} \leq x_{T}^{u}$ & $x_{T}^{u} \leq x_{T-1} \leq x_{T}^{d}$ \\
\hline Corta & $x_{T}^{u} \leq x_{T-1} \leq x_{T}^{d}$ & $x_{T}^{d} \leq x_{T-1} \leq x_{T}^{u}$ \\
\hline
\end{tabular}

Fuente: elaboración propia. 
Las desigualdades de la tabla 1 basan su desarrollo en el siguiente análisis:

Opción call: desde la perspectiva del comprador de la opción (posición larga) que prevé un escenario alcista para los precios del activo subyacente, se asume que la materialización de este en cada periodo aumentará la probabilidad de estar in the money al momento del ejercicio y, consecuentemente, de efectuar la compra del activo motivando un ajuste creciente a la posición del activo riesgoso. En contraste, para la posición corta en la opción, cada vez que se materialice un incremento de los precios, será más probable que llegado el momento de ejercicio se obligue a vender el activo lo que motivará un ajuste decreciente de la posición.

Opción put: detrás de la posición larga en una opción de venta se encuentran las expectativas bajistas frente al precio del activo subyacente, lo cual hace que en el caso de una caída de precios en cada periodo se refleje una tendencia a que se ejerza el derecho de venta, de esta manera la cantidad de activo riesgoso se verá reducida. Caso contrario sucede con la posición inversa, ya que el vendedor de la opción será quien compre el activo subyacente del instrumento financiero.

Precio opción europea larga de compra (long call). Aplicando la desigualdad a las ecuaciones (23) y (24) se reescriben las ecuaciones de la siguiente forma:

$$
\begin{aligned}
& x_{T-1} S_{T-1} u+B_{0}\left(1+r_{f}\right)^{T-1}=Z_{T}^{u}+k_{p}\left(x_{T}^{u}-x_{T-1}\right) u S_{T-1} \\
& x_{T-1} S_{T-1} d+B_{0}\left(1+r_{f}\right)^{T-1}=Z_{T}^{d}+k_{p}\left(x_{T-1}-x_{T}^{d}\right) d S_{T-1}
\end{aligned}
$$

De las ecuaciones (28) y (29) obtenemos que:

$$
\begin{gathered}
y_{T-1}=\frac{Z_{T}^{u}-Z_{T}^{d}+k_{P} S_{T-1}\left(u x_{T}^{u}+d x_{T}^{d}\right)}{(\bar{u}-\bar{d}) S_{T-1}} \\
y_{T-1}=\frac{\bar{u} Z_{T}^{d}-\bar{d} Z_{T}^{u}-\left[k_{P} S_{T-1}\left(u \bar{d} x_{T}^{u}+d \bar{u} x_{T}^{d}\right)\right]}{(\bar{u}-\bar{d}) S_{T-1}}
\end{gathered}
$$


Donde: $\bar{u}=u\left(1+k_{P}\right)$ y $\bar{d}=d\left(1-k_{P}\right)$

$\mathrm{Y}$ donde $Z_{T}=\max \left\{S_{T}-K ; 0\right\}$

Para obtener el valor de la opción es necesario efectuar un proceso iterativo desde la fecha de vencimiento de la opción hasta el tiempo inicial $t=0$, a través de la composición del portafolio que la replica en cada nodo. Se obtienen así las cantidades tanto de activo riesgoso y libre de riesgo, una vez se cubran los costos de transacción causados en el rebalanceo.

Es importante resaltar que, en el caso in the money, el portafolio que replica el valor de la Opción Europea Larga de Compra estará compuesto por una unidad de activo riesgoso y una posición corta en el activo libre de riesgo igual al precio del ejercicio.

Precio opción europea corta de compra (short call). Replicando el ejercicio anterior se obtienen las siguientes ecuaciones:

$$
\begin{aligned}
& x_{T-1} S_{T-1} u+B_{0}\left(1+r_{f}\right)^{T-1}=Z_{T}^{u}+k_{P}\left(x_{T-1}-x_{T}^{u}\right) u S_{T-1} \\
& x_{T-1} S_{T-1} d+B_{0}\left(1+r_{f}\right)^{T-1}=Z_{T}^{d}+k_{P}\left(x_{T}^{d}-x_{T-1}\right) d S_{T-1}
\end{aligned}
$$

De las ecuaciones 30 y 31 se obtiene que:

$$
\begin{gathered}
x_{T-1}=\frac{Z_{T}^{u}-Z_{T}^{d}-k_{P} S_{T-1}\left(u x_{T}^{u}+d x_{T}^{d}\right)}{(\bar{u}-\bar{d}) S_{T-1}} \\
y_{T-1}=\frac{\bar{u} Z_{T}^{d}-\bar{d} Z_{T}^{u}+\left[k_{P} S_{T-1}\left(u \bar{d} x_{T}^{u}+d \bar{u} x_{T}^{d}\right)\right]}{(\bar{u}-\bar{d}) S_{T-1}}
\end{gathered}
$$

Donde: $\bar{u}=u\left(1-k_{P}\right)$ y $\bar{d}=d\left(1+k_{P}\right)$

Y donde: $Z_{T}=\max \left\{S_{T}-K ; 0\right\}$ 
En el caso en que la opción esté in the money, el tenedor del derivado ejercerá su derecho; de esta manera, la composición del portafolio de réplica queda con una posición corta en el activo riesgoso, más una posición larga en el activo libre de riesgo por el valor del precio del ejercicio.

Precio opción europea larga de venta (long put). Al igual que el ejercicio realizado con las opciones europeas de compra, las opciones europeas de venta parten de la aplicación de ecuaciones 23 y 24 junto a las desigualdades de la tabla 1. Se obtienen las siguientes ecuaciones:

$$
\begin{aligned}
& x_{T-1} S_{T-1} u+B_{0}\left(1+r_{f}\right)^{T-1}=Z_{T}^{u}+k_{P}\left(x_{T}^{u}-x_{T-1}\right) u S_{T-1} \\
& x_{T-1} S_{T-1} d+B_{0}\left(1+r_{f}\right)^{T-1}=Z_{T}^{d}+k_{P}\left(x_{T-1}-x_{T}^{d}\right) d S_{T-1}
\end{aligned}
$$

De las ecuaciones 33 y 34 se obtiene que:

$$
\begin{gathered}
x_{T-1}=\frac{Z_{T}^{u}-Z_{T}^{d}+k_{P} S_{T-1}\left(u x_{T}^{u}+d x_{T}^{d}\right)}{(\bar{u}-\bar{d}) S_{T-1}} \\
x_{T-1}=\frac{\bar{u} Z_{T}^{d}-\bar{d} Z_{T}^{u}+\left[k_{P} S_{T-1}\left(u \bar{d} x_{T}^{u}+d \bar{u} x_{T}^{d}\right)\right]}{(\bar{u}-\bar{d}) S_{T-1}}
\end{gathered}
$$

Donde: $\bar{u}=u\left(1+k_{P}\right)$ y $\bar{d}=d\left(1-k_{P}\right)$

Y donde: $Z_{T}=\max \left\{K-S_{T} ; 0\right\}$

En el caso in the money, el tenedor ejercerá el derecho de vender el subyacente, y generará un portafolio de réplica compuesto por una unidad en corto del activo riesgoso, más una posición corta en el activo libre de riesgo equivalente al precio de ejercicio de la opción.

Precio opción europea corta de venta (short put). Finalmente, en el caso del vendedor de la opción de venta, aplicando el mismo ejercicio realizado anteriormente, se obtienen las siguientes ecuaciones lineales: 


$$
\begin{aligned}
& x_{T-1} S_{T-1} u+B_{0}\left(1+r_{f}\right)^{T-1}=Z_{T}^{u}+k_{P}\left(x_{T-1}-x_{T}^{u}\right) u S_{T-1} \\
& x_{T-1} S_{T-1} d+B_{0}\left(1+r_{f}\right)^{T-1}=Z_{T}^{d}+k_{P}\left(x_{T}^{d}-x_{T-1}\right) d S_{T-1}
\end{aligned}
$$

De las ecuaciones 40 y 41 se obtiene que:

$$
\begin{gathered}
x_{T-1}=\frac{Z_{T}^{u}-Z_{T}^{d} k_{P} S_{T-1}\left(u x_{T}^{u}+d x_{T}^{d}\right)}{(\bar{u}-\bar{d}) S_{T-1}} \\
y_{T-1}=\frac{\bar{u} Z_{T}^{d}-\bar{d} Z_{T}^{u}\left[k_{P} S_{T-1}\left(u \bar{d} x_{T}^{u}+d \bar{u} x_{T}^{d}\right)\right]}{(\bar{u}-\bar{d}) S_{T-1}}
\end{gathered}
$$

Donde: $\bar{u}=u\left(1-k_{P}\right)$ y $\bar{d}=d\left(1+k_{P}\right)$

Y donde: $Z_{T}=\max \left\{K-S_{T} ; 0\right\}$

Producto de la naturaleza de este tipo de opción, la composición del portafolio de réplica en el vencimiento consistirá en una posición larga en el activo riesgoso y una posición corta en el activo libre de riesgo, en el caso en que la opción esté in the money, ya que el comprador ejercerá la opción de venta.

Modelo general de valoración de opciones europeas con costos de transacción proporcionales. A continuación se presenta el modelo generalizado para la valoración de opciones europeas con costos de transacción, en este se resumen las posibles variaciones que se describieron anteriormente, las cuales pueden presentarse tanto por el tipo de opción como por su posición.

Modelo general:

$$
\begin{gathered}
x_{T-1}=\frac{Z_{T}^{u}-Z_{T}^{d}+\left[\lambda k_{P} S_{T-1}\left(u x_{T}^{u}+d x_{T}^{d}\right)\right]}{(\bar{u}-\bar{d}) S_{T-1}} \\
y_{T-1}=\frac{\bar{u} Z_{T}^{d}-\bar{d} Z_{T}^{u}-\left[\lambda k_{P} S_{T-1}\left(u \bar{d} x_{T}^{u}+d \bar{u} x_{T}^{d}\right)\right]}{(\bar{u}-\bar{d}) S_{T-1}}
\end{gathered}
$$


Donde: $\bar{u}=u\left(1+\lambda k_{P}\right)$ y $\bar{d}=d\left(1-\lambda k_{P}\right)$

De esta forma, siguiendo la metodología de construcción del portafolio de replicación de la opción se generan las ecuaciones (45), (46) y (47) para el cálculo de las cantidades de activo riesgoso y libre de riesgo respectivamente; asimismo, se presenta la variable indicadora $\lambda$ la cual introduce al modelo las variaciones descritas, de la siguiente manera:

$$
\lambda=\left\{\begin{array}{l}
1, \text { posición larga call y larga put } \\
-1, \text { posición corta call y corta put }
\end{array}\right.
$$

\subsubsection{Modelo con costos de transacción proporcionales $\left(k_{p}\right)$ más fijos $\left(k_{f}\right)$}

Este modelo incorpora los costos de transacción tanto proporcionales como fijos, estos últimos se asumirán como la comisión que se causa a favor del creador de mercado por garantizar a los inversionistas las cantidades y las posiciones en cada uno de los puntos de revisión, es decir, como un costo de liquidez sobre el activo subyacente.

Al contemplar la coexistencia de los dos costos es posible inferir que: i) la combinación de costos de transacción fijos y proporcionales hará que el ajuste de posiciones sea más costoso y, consecuentemente, el valor de la opción; y ii) los costos fijos son indiferentes al monto comercializado, lo cual conlleva un desestímulo a las pequeñas transacciones de activo riesgoso.

Ahora, partiendo de las ecuaciones (23) y (24) se llega a dos nuevas ecuaciones no lineales:

$$
\begin{aligned}
& x_{T-1} S u+B_{0}\left(1+r_{f}\right)^{T-1}=Z_{T}^{u} k_{P}\left|x_{T-1}-x_{T}^{u}\right| u S+k_{f}^{+} \\
& x_{T-1} S d+B_{0}\left(1+r_{f}\right)^{T-1}=Z_{T}^{d} k_{P}\left|x_{T-1}-x_{T}^{d}\right| d S+k_{f}^{-}
\end{aligned}
$$

Para este caso, al segundo miembro de la ecuación no lineal se suma la provisión para cubrir tanto los costos proporcionales como los fijos. De esta manera, el valor del portafolio, al ser autofinanciado, en cualquiera de los dos estados que se presente, alcanzará para efectuar el ajuste de las posiciones y cubrir los costos derivados del mismo. 
Modelo general de valoración de opciones europeas con costos de transacción proporcionales $\left(\boldsymbol{k}_{p}\right)$ más fijos $\left(\boldsymbol{k}_{f}\right)$. Este modelo se construye siguiendo los mismos fundamentos desarrollados durante la construcción del modelo generalizado con costos de transacción proporcionales, presentando una extensión donde se incluyen los costos fijos como porcentaje del Spot. A partir de esto se obtiene la siguiente forma general del modelo:

$$
\begin{gathered}
x_{T-1}=\frac{Z_{T}^{u}-Z_{T}^{d}+\left[\lambda k_{p} S_{T-1}\left(u x_{T}^{u}+d x_{T}^{d}\right)\right]+2 S_{0} \varpi k_{f}}{(\bar{u}-\bar{d}) S_{T-1}} \\
y_{T-1}=\frac{\bar{u} Z_{T}^{d}-\bar{d} Z_{T}^{u}+\left[\lambda k_{p} S_{T-1}\left(u \bar{d} x_{T}^{u}+d \bar{u} x_{T}^{d}\right)\right]+\left[\bar{u} k_{f}^{-}-\bar{d} k_{f}^{+}\right]}{(\bar{u}-\bar{d}) S_{T-1}}
\end{gathered}
$$

Donde: $\bar{u}=u\left(1+\lambda k_{P}\right), \bar{d}=d\left(1-\lambda k_{P}\right), k_{f}^{+}=S_{0}\left(1-\varpi k_{f}\right), k_{f}^{-}=S_{0}\left(1+\varpi k_{f}\right)$ $\mathrm{y}$

$$
\lambda=\left\{\begin{array}{l}
1, \text { posición larga call y larga put } \\
-1, \text { posición corta call y corta } p u t
\end{array}\right.
$$

En este modelo se genera una variable indicadora $\varpi$ que, al igual que $\lambda$ hace que el modelo pueda generalizar la valoración de opciones de compra y venta desde la perspectiva, ya sea del tenedor de la opción o del vendedor.

$$
\varpi=\left\{\begin{array}{l}
1, \text { posición larga call y larga put } \\
-1, \text { posición corta call y corta put }
\end{array}\right.
$$

A partir de las ecuaciones (51) y (52) el modelo calculará, durante la inducción hacia atrás, el monto invertido en activo riesgoso $\left(x_{t} S_{t}\right)$ y el monto invertido en libre de riesgo $y_{t}$ para cada nodo, lo anterior para replicar el valor de la opción europea a través de la expresión (3) que describe la estructura del portafolio.

La generalización de este modelo permitirá, no solo definir a través de las variables indicadoras $\varpi$ y $\lambda$ si se trata de una opción call o put en su posición larga o corta, sino incluir tanto costos de transacción proporcionales como fijos, 
haciendo a $k_{p}>0$ y $k_{f}>0$ respectivamente. Por el contrario, si $k_{f}=0$ y $k_{p}=0$ se replicará el modelo CRR original.

Extensión del modelo general de valoración de opciones europeas para la valoración de opciones americanas. En esta sección se realizará la extensión del modelo, incluyendo la posibilidad que tiene el tenedor de la opción de elegir el mayor beneficio entre realizar el ejercicio anticipado (payoff) o continuar con la opción un periodo más, entendiendo esto último como el valor dado por el modelo general para cada nodo en el caso europeo a través de las ecuaciones (51) y (52), y que denominaremos valor de continuidad. Lo anterior se puede expresar de la siguiente forma (Rogers y Talay, 1999, pp. 49):

Call: $\max \left\{\left(S_{T-1}-K\right)^{+} ;\left(x_{T-1} S_{T-1}\right)+y_{T-1}\right\}$

Put: $\max \{\underbrace{\left(S_{T-1}-K\right)^{-}}_{\text {Payoff }} ; \underbrace{\left(x_{T-1} S_{T-1}\right)+y_{T-1}}_{\text {Valor de continuidad }}\}$

Las expresiones 55 y 56 muestran cómo para la replicación de una opción americana, el valor de continuidad se expresa de forma general como $\left\{\left(x_{t} S_{t}\right)+y_{t}\right\}$, siendo $x_{t}$ y $y_{t}$ los valores calculados a partir de las ecuaciones (51) y (52), respectivamente.

De esta manera, en caso de ser ejercida la opción anticipadamente, las posiciones de activo riesgoso y libre de riesgo que tomará el denominado portafolio principal serán las mismas definidas en el vencimiento de la opción europea, las cuales se resumen a continuación:

Tabla 2: Posiciones de activo riesgoso y libre de riesgo cuando se ejerce la opción

\begin{tabular}{|c|c|c|c|}
\hline \multirow{2}{*}{ Opción } & Posición & Activo riesgoso & Activo libre de riesgo \\
\hline \multirow{2}{*}{ Call } & Larga & Posición larga & Posición corta \\
\cline { 2 - 4 } & Corta & Posición corta & Posición larga \\
\hline \multirow{2}{*}{ Put } & Larga & Posición corta & Posición larga \\
\cline { 2 - 4 } & Corta & Posición larga & Posición corta \\
\hline
\end{tabular}

Fuente: elaboración propia. 


\section{Resultados de la implementación de los modelos}

Con el objetivo de revisar los impactos derivados de la inclusión de los costos de transacción proporcionales y fijos, en esta sección se implementan los modelos generales presentados en el capítulo anterior. Se obtienen así los resultados consignados en las secciones 3.1. y 3.2.

En la tabla 3 se relacionan los datos que se consideraron para la obtención de resultados.

Tabla 3: Datos para la simulación

\begin{tabular}{|c|c|c|}
\hline Factores & Datos $k_{p}$ & Datos $k f$ \\
\hline Spot & USD 100 & USD 100 \\
\hline Strike & USD $80 ; 90 ; 100 ; 110 ; 120$ & USD 80; $90 ; 100 ; 110 ; 120$ \\
\hline Número de pasos & $6 ; 13 ; 52 ; 150$ & $6 ; 13 ; 52 ; 150$ \\
\hline Costos proporcionales $(\%)$ & $0 ; 0,125 ; 0,5 ; 2$ & $0 ; 0,125 ; 0,5 ; 2$ \\
\hline Costos fijos $(\%)$ & 0 & 0,125 \\
\hline
\end{tabular}

Fuente: elaboración propia.

\subsection{Costos proporcionales}

Tabla 4: Resultados valoración opción europea larga de venta (costos proporcionales)

Opción europea larga de venta (long put)

\begin{tabular}{|c|c|c|c|c|}
\hline \multirow{2}{*}{ Strike } & \multicolumn{4}{|c|}{ Número de nodos (n) } \\
\cline { 2 - 5 } & $\mathbf{6}$ & $\mathbf{1 3}$ & $\mathbf{5 2}$ & $\mathbf{1 5 0}$ \\
\hline 80 & 0,430 & 0,428 & 0,392 & 0,402 \\
\hline 90 & 1,640 & 1,558 & 1,485 & 1,494 \\
\hline 100 & 3,564 & 4,002 & 3,862 & 3,888 \\
\hline 110 & 8,129 & 8,026 & 7,972 & 7,954 \\
\hline 120 & 13,307 & 13,518 & 13,639 & 13,648 \\
\hline & & $\mathrm{k}=0,125 \%$ & $\mathbf{1 5}$ \\
\hline Strike & $\mathbf{6}$ & $\mathbf{1 3}$ & $\mathbf{5 2}$ & $\mathbf{1 5 0}$ \\
\hline 80 & 0,463 & 0,474 & 0,480 & 0,556 \\
\hline
\end{tabular}




\begin{tabular}{|c|c|c|c|c|}
\hline 90 & 1,713 & 1,661 & 1,683 & 1,827 \\
\hline 100 & 3,679 & 4,157 & 4,166 & 4,393 \\
\hline 110 & 8,254 & 8,205 & 8,324 & 8,542 \\
\hline 120 & 13,420 & 13,686 & 13,973 & 14,208 \\
\hline \multicolumn{4}{|c|}{$\mathrm{k}=0,5 \%$} \\
\hline Strike & $\mathbf{6}$ & $\mathbf{1 3}$ & $\mathbf{5 2}$ & $\mathbf{1 5 0}$ \\
\hline 80 & 0,564 & 0,621 & 0,774 & 1,083 \\
\hline 90 & 1,931 & 1,967 & 2,271 & 2,803 \\
\hline 100 & 4,017 & 4,608 & 5,021 & 5,762 \\
\hline 110 & 8,618 & 8,721 & 9,300 & 10,101 \\
\hline 120 & 13,753 & 14,175 & 14,911 & 15,722 \\
\hline & & $\mathrm{k}=2 \%$ & $\mathbf{1 5}$ \\
\hline Strike & $\mathbf{6}$ & $\mathbf{1 3}$ & $\mathbf{5 2}$ & $\mathbf{1 5 0}$ \\
\hline 80 & 1,024 & 1,290 & 2,136 & 3,424 \\
\hline 90 & 2,801 & 3,164 & 4,462 & 6,214 \\
\hline 100 & 5,277 & 6,242 & 7,877 & 10,000 \\
\hline 110 & 9,965 & 10,554 & 12,469 & 14,760 \\
\hline 120 & 15,017 & 15,950 & 18,041 & 20,388 \\
\hline
\end{tabular}

Fuente: elaboración propia.

Tabla 5: Resultados valoración opción europea corta de venta (costos proporcionales)

Opción europea corta de venta (short put)

\begin{tabular}{|c|c|c|c|c|}
\hline \multirow{2}{*}{} & \multicolumn{4}{|c|}{ Número de nodos (n) } \\
\cline { 2 - 5 } Strike & $\mathbf{6}$ & $\mathbf{1 3}$ & $\mathbf{5 2}$ & $\mathbf{1 5 0}$ \\
\hline 80 & 0,430 & 0,428 & 0,392 & 0,402 \\
\hline 90 & 1,640 & 1,558 & 1,485 & 1,494 \\
\hline 100 & 3,564 & 4,002 & 3,862 & 3,888 \\
\hline 110 & 8,129 & 8,026 & 7,972 & 7,954 \\
\hline 120 & 13,307 & 13,518 & 13,639 & 13,648 \\
\hline & \multicolumn{4}{|c|}{$\mathrm{k}=0,125 \%$} \\
\hline
\end{tabular}




\begin{tabular}{|c|c|c|c|c|}
\hline Strike & 6 & 13 & 52 & 150 \\
\hline 80 & 0,398 & 0,384 & 0,309 & 0,265 \\
\hline 90 & 1,567 & 1,456 & 1,287 & 1,162 \\
\hline 100 & 3,448 & 3,844 & 3,548 & 3,352 \\
\hline 110 & 8,003 & 7,843 & 7,604 & 7,319 \\
\hline \multirow[t]{2}{*}{120} & 13,193 & 13,347 & 13,293 & 13,052 \\
\hline & \multicolumn{4}{|c|}{$\mathrm{k}=0,5 \%$} \\
\hline Strike & 6 & 13 & 52 & 150 \\
\hline 80 & 0,309 & 0,261 & 0,111 & 0,017 \\
\hline 90 & 1,349 & 1,151 & 0,708 & 0,252 \\
\hline 100 & 3,091 & 3,354 & 2,526 & 1,487 \\
\hline 110 & 7,614 & 7,269 & 6,374 & 4,942 \\
\hline \multirow[t]{2}{*}{120} & 12,845 & 12,817 & 12,168 & 10,961 \\
\hline & \multicolumn{4}{|c|}{$\mathrm{k}=2 \%$} \\
\hline Strike & 6 & 13 & 52 & 150 \\
\hline 80 & 0,054 & 0,003 & $*$ & $*$ \\
\hline 90 & 0,515 & 0,099 & $*$ & $*$ \\
\hline 100 & 1,523 & 1,024 & $*$ & $*$ \\
\hline 110 & 5,845 & 4,311 & $*$ & $*$ \\
\hline 120 & 11,360 & 10,376 & $*$ & $*$ \\
\hline
\end{tabular}

Fuente: elaboración propia.

Tabla 6: Resultados valoración opción americana larga de venta (costos proporcionales)

Opción americana larga de venta (long put)

\begin{tabular}{|c|c|c|c|c|}
\hline \multirow{2}{*}{ Strike } & \multicolumn{4}{|c|}{ Número de nodos (n) } \\
\cline { 2 - 5 } & $\mathbf{6}$ & $\mathbf{1 3}$ & $\mathbf{5 2}$ & $\mathbf{1 5 0}$ \\
\hline 80 & 0,457 & 0,472 & 0,450 & 0,458 \\
\hline 90 & 1,881 & 1,805 & 1,774 & 1,777 \\
\hline 100 & 4,747 & 4,937 & 4,903 & 4,913 \\
\hline 110 & 10,685 & 10,826 & 10,803 & 10,798 \\
\hline 120 & 18,294 & 19,123 & 19,780 & 19,924 \\
\hline & & $\mathrm{k}=0,125 \%$ & \\
\hline
\end{tabular}




\begin{tabular}{|c|c|c|c|c|}
\hline Strike & $\mathbf{6}$ & $\mathbf{1 3}$ & $\mathbf{5 2}$ & $\mathbf{1 5 0}$ \\
\hline 80 & 0,491 & 0,522 & 0,548 & 0,629 \\
\hline 90 & 1,962 & 1,917 & 1,994 & 2,144 \\
\hline 100 & 4,860 & 5,104 & 5,207 & 5,429 \\
\hline 110 & 10,788 & 10,948 & 11,034 & 11,202 \\
\hline 120 & 18,367 & 19,168 & 19,780 & 19,924 \\
\hline & \multicolumn{5}{|c|}{$\mathrm{k}=0,5 \%$} \\
\hline Strike & $\mathbf{6}$ & $\mathbf{1 3}$ & $\mathbf{5 2}$ & $\mathbf{1 5 0}$ \\
\hline 80 & 0,596 & 0,682 & 0,869 & 1,205 \\
\hline 90 & 2,204 & 2,253 & 2,639 & 3,202 \\
\hline 100 & 5,191 & 5,582 & 6,072 & 6,821 \\
\hline 110 & 11,087 & 11,300 & 11,768 & 12,425 \\
\hline 120 & 18,600 & 19,385 & 19,972 & 20,236 \\
\hline \multicolumn{5}{|c|}{$\mathrm{k}=2 \%$} \\
\hline Strike & $\mathbf{6}$ & $\mathbf{1 3}$ & $\mathbf{5 2}$ & $\mathbf{1 5 0}$ \\
\hline 80 & 1,070 & 1,407 & 2,329 & 3,700 \\
\hline 90 & 3,115 & 3,547 & 4,984 & 6,796 \\
\hline 100 & 6,400 & 7,262 & 8,960 & 11,114 \\
\hline 110 & 12,247 & 12,850 & 14,510 & 16,648 \\
\hline 120 & 19,567 & 20,330 & 21,581 & 23,394 \\
\hline
\end{tabular}

Fuente: elaboración propia.

Tabla 7: Resultados valoración opción americana corta de venta (costos proporcionales)

Opción americana corta de venta (short put)

\begin{tabular}{|c|c|c|c|c|}
\hline \multirow{2}{*}{} & \multicolumn{4}{|c|}{ Número de nodos (n) } \\
\cline { 2 - 5 } Strike & $\mathbf{6}$ & $\mathbf{1 3}$ & $\mathbf{5 2}$ & $\mathbf{1 5 0}$ \\
\hline 80 & 0,457 & 0,472 & 0,450 & 0,458 \\
\hline 90 & 1,881 & 1,805 & 1,774 & 1,777 \\
\hline 100 & 4,747 & 4,937 & 4,903 & 4,913 \\
\hline 110 & 10,685 & 10,826 & 10,803 & 10,798 \\
\hline 120 & 18,294 & 19,123 & 19,780 & 19,924 \\
\hline & \multicolumn{4}{|c|}{$\mathrm{k}=0,125 \%$} \\
\hline
\end{tabular}




\begin{tabular}{|c|c|c|c|c|}
\hline Strike & 6 & 13 & 52 & 150 \\
\hline 80 & 0,424 & 0,424 & 0,359 & 0,305 \\
\hline 90 & 1,800 & 1,694 & 1,553 & 1,404 \\
\hline 100 & 4,632 & 4,770 & 4,584 & 4,363 \\
\hline 110 & 10,580 & 10,701 & 10,558 & 10,422 \\
\hline \multirow[t]{2}{*}{120} & 18,221 & 19,123 & 19,780 & 19,924 \\
\hline & \multicolumn{4}{|c|}{$\mathrm{k}=0,5 \%$} \\
\hline Strike & 6 & 13 & 52 & 150 \\
\hline 80 & 0,331 & 0,289 & 0,135 & 0,021 \\
\hline 90 & 1,555 & 1,358 & 0,890 & 0,338 \\
\hline 100 & 4,275 & 4,276 & 3,540 & 2,401 \\
\hline 110 & 10,231 & 10,304 & 9,993 & 9,930 \\
\hline \multirow[t]{2}{*}{120} & 18,109 & 19,123 & 19,780 & 19,924 \\
\hline & \multicolumn{4}{|c|}{$\mathrm{k}=2 \%$} \\
\hline Strike & 6 & 13 & 52 & 150 \\
\hline 80 & 0,059 & 0,004 & $*$ & $*$ \\
\hline 90 & 0,546 & 0,138 & $*$ & $*$ \\
\hline 100 & 2,907 & 1,852 & $*$ & $*$ \\
\hline 110 & 9,045 & 9,196 & $*$ & $*$ \\
\hline 120 & 18,109 & 19,123 & $*$ & $*$ \\
\hline
\end{tabular}

Fuente: elaboración propia.

Tabla 8: Resultados valoración opción europea larga de compra (costos proporcionales)

Opción europea larga de compra (long call)

\begin{tabular}{|c|c|c|c|c|}
\hline \multirow{2}{*}{ Strike } & \multicolumn{4}{|c|}{ Número de nodos (n) } \\
\cline { 2 - 5 } & $\mathbf{6}$ & $\mathbf{1 3}$ & $\mathbf{5 2}$ & $\mathbf{1 5 0}$ \\
\hline 80 & 27,703 & 27,701 & 27,665 & 27,675 \\
\hline 90 & 19,821 & 19,740 & 19,667 & 19,676 \\
\hline 100 & 12,655 & 13,093 & 12,953 & 12,979 \\
\hline 110 & 8,129 & 8,026 & 7,972 & 7,954 \\
\hline 120 & 4,216 & 4,427 & 4,548 & 4,557 \\
\hline & \multicolumn{5}{|c|}{$\mathrm{k}=0,125 \%$} \\
\hline
\end{tabular}




\begin{tabular}{|c|c|c|c|c|}
\hline Strike & 6 & 13 & 52 & 150 \\
\hline 80 & 27,735 & 27,747 & 27,753 & 27,829 \\
\hline 90 & 19,894 & 19,842 & 19,865 & 20,009 \\
\hline 100 & 12,770 & 13,248 & 13,256 & 13,484 \\
\hline 110 & 8,254 & 8,205 & 8,324 & 8,542 \\
\hline \multirow[t]{2}{*}{120} & 4,329 & 4,595 & 4,882 & 5,117 \\
\hline & \multicolumn{4}{|c|}{$\mathrm{k}=0,5 \%$} \\
\hline Strike & 6 & 13 & 52 & 150 \\
\hline 80 & 27,837 & 27,894 & 28,047 & 28,356 \\
\hline 90 & 20,113 & 20,149 & 20,453 & 20,985 \\
\hline 100 & 13,107 & 13,699 & 14,111 & 14,853 \\
\hline 110 & 8,618 & 8,721 & 9,300 & 10,101 \\
\hline \multirow[t]{2}{*}{120} & 4,663 & 5,084 & 5,820 & 6,632 \\
\hline & \multicolumn{4}{|c|}{$\mathrm{k}=2 \%$} \\
\hline Strike & 6 & 13 & 52 & 150 \\
\hline 80 & 28,297 & 28,563 & 29,409 & 30,696 \\
\hline 90 & 20,983 & 21,346 & 22,643 & 24,396 \\
\hline 100 & 14,368 & 15,333 & 16,967 & 19,091 \\
\hline 110 & 9,965 & 10,554 & 12,469 & 14,760 \\
\hline 120 & 5,926 & 6,859 & 8,950 & 11,297 \\
\hline
\end{tabular}

Fuente: elaboración propia.

Tabla 9: Resultados valoración opción europea corta de compra (costos proporcionales)

Opción europea corta de compra (short call)

\begin{tabular}{|c|c|c|c|c|}
\hline \multirow{2}{*}{} & \multicolumn{4}{|c|}{ Número de nodos (n) } \\
\cline { 2 - 5 } Strike & $\mathbf{6}$ & $\mathbf{1 3}$ & $\mathbf{5 2}$ & $\mathbf{1 5 0}$ \\
\hline 80 & 27,703 & 27,701 & 27,665 & 27,675 \\
\hline 90 & 19,821 & 19,740 & 19,667 & 19,676 \\
\hline 100 & 12,655 & 13,093 & 12,953 & 12,979 \\
\hline 110 & 8,129 & 8,026 & 7,972 & 7,954 \\
\hline 120 & 4,216 & 4,427 & 4,548 & 4,557 \\
\hline & \multicolumn{4}{|c|}{$\mathrm{k}=0,125 \%$} \\
\hline
\end{tabular}




\begin{tabular}{|c|c|c|c|c|}
\hline Strike & $\mathbf{6}$ & $\mathbf{1 3}$ & $\mathbf{5 2}$ & $\mathbf{1 5 0}$ \\
\hline 80 & 27,671 & 27,656 & 27,582 & 27,538 \\
\hline 90 & 19,749 & 19,638 & 19,469 & 19,343 \\
\hline 100 & 12,539 & 12,935 & 12,638 & 12,443 \\
\hline 110 & 8,003 & 7,843 & 7,604 & 7,319 \\
\hline 120 & 4,102 & 4,256 & 4,202 & 3,961 \\
\hline & \multicolumn{5}{|c|}{$\mathrm{k}=0,5 \%$} \\
\hline Strike & $\mathbf{6}$ & $\mathbf{1 3}$ & $\mathbf{5 2}$ & $\mathbf{1 5 0}$ \\
\hline 80 & 27,582 & 27,534 & 27,384 & 27,290 \\
\hline 90 & 19,531 & 19,333 & 18,889 & 18,434 \\
\hline 100 & 12,182 & 12,445 & 11,617 & 10,578 \\
\hline 110 & 7,614 & 7,269 & 6,374 & 4,942 \\
\hline 120 & 3,754 & 3,726 & 3,077 & 1,870 \\
\hline \multicolumn{5}{|c|}{$\mathrm{k}=2 \%$} \\
\hline Strike & $\mathbf{6}$ & $\mathbf{1 3}$ & $\mathbf{5 2}$ & $\mathbf{1 5 0}$ \\
\hline 80 & 27,327 & 27,276 & $*$ & $*$ \\
\hline 90 & 18,697 & 18,281 & $*$ & $*$ \\
\hline 100 & 10,614 & 10,115 & $*$ & $*$ \\
\hline 110 & 5,845 & 4,311 & $*$ & $*$ \\
\hline 120 & 2,269 & 1,285 & $*$ & $*$ \\
\hline
\end{tabular}

Fuente: elaboración propia.

Tabla 10: Resultados valoración opción americana larga de compra (costos proporcionales)

Opción americana larga de compra (long call)

\begin{tabular}{|c|c|c|c|c|}
\hline \multirow{2}{*}{} & \multicolumn{4}{|c|}{ Número de nodos (n) } \\
\cline { 2 - 5 } Strike & $\mathbf{6}$ & $\mathbf{1 3}$ & $\mathbf{5 2}$ & $\mathbf{1 5 0}$ \\
\hline 80 & 27,703 & 27,701 & 27,665 & 27,675 \\
\hline 90 & 19,821 & 19,740 & 19,667 & 19,676 \\
\hline 100 & 12,655 & 13,093 & 12,953 & 12,979 \\
\hline 110 & 8,129 & 8,026 & 7,972 & 7,954 \\
\hline 120 & 4,216 & 4,427 & 4,548 & 4,557 \\
\hline & \multicolumn{4}{|c|}{$\mathrm{k}=0,125 \%$} \\
\hline
\end{tabular}




\begin{tabular}{|c|c|c|c|c|}
\hline Strike & $\mathbf{6}$ & $\mathbf{1 3}$ & $\mathbf{5 2}$ & $\mathbf{1 5 0}$ \\
\hline 80 & 27,735 & 27,747 & 27,753 & 27,829 \\
\hline 90 & 19,894 & 19,842 & 19,865 & 20,009 \\
\hline 100 & 12,770 & 13,248 & 13,256 & 13,484 \\
\hline 110 & 8,254 & 8,205 & 8,324 & 8,542 \\
\hline 120 & 4,329 & 4,595 & 4,882 & 5,117 \\
\hline & \multicolumn{5}{|c|}{$\mathrm{k}=0,5 \%$} \\
\hline Strike & $\mathbf{6}$ & $\mathbf{1 3}$ & $\mathbf{5 2}$ & $\mathbf{1 5 0}$ \\
\hline 80 & 27,837 & 27,894 & 28,047 & 28,356 \\
\hline 90 & 20,113 & 20,149 & 20,453 & 20,985 \\
\hline 100 & 13,107 & 13,699 & 14,111 & 14,853 \\
\hline 110 & 8,618 & 8,721 & 9,300 & 10,101 \\
\hline 120 & 4,663 & 5,084 & 5,820 & 6,632 \\
\hline \multicolumn{5}{|c|}{$\mathrm{k}=2 \%$} \\
\hline Strike & $\mathbf{6}$ & $\mathbf{1 3}$ & $\mathbf{5 2}$ & $\mathbf{1 5 0}$ \\
\hline 80 & 28,297 & 28,563 & 29,409 & 30,696 \\
\hline 90 & 20,983 & 21,346 & 22,643 & 24,396 \\
\hline 100 & 14,368 & 15,333 & 16,967 & 19,091 \\
\hline 110 & 9,965 & 10,554 & 12,469 & 14,760 \\
\hline 120 & 5,926 & 6,859 & 8,950 & 11,297 \\
\hline
\end{tabular}

Fuente: elaboración propia.

Tabla 11: Resultados valoración opción americana corta de compra (costos proporcionales)

Opción americana corta de compra (short call)

\begin{tabular}{|c|c|c|c|c|}
\hline \multirow{2}{*}{} & \multicolumn{4}{|c|}{ Número de nodos (n) } \\
\cline { 2 - 5 } Strike & $\mathbf{6}$ & $\mathbf{1 3}$ & $\mathbf{5 2}$ & $\mathbf{1 5 0}$ \\
\hline 80 & 27,703 & 27,701 & 27,665 & 27,675 \\
\hline 90 & 19,821 & 19,740 & 19,667 & 19,676 \\
\hline 100 & 12,655 & 13,093 & 12,953 & 12,979 \\
\hline 110 & 8,129 & 8,026 & 7,972 & 7,954 \\
\hline 120 & 4,216 & 4,427 & 4,548 & 4,557 \\
\hline & \multicolumn{5}{|c|}{$\mathrm{k}=0,125 \%$} \\
\hline
\end{tabular}




\begin{tabular}{|c|c|c|c|c|}
\hline Strike & 6 & 13 & 52 & 150 \\
\hline 80 & 27,671 & 27,656 & 27,582 & 27,538 \\
\hline 90 & 19,749 & 19,638 & 19,469 & 19,343 \\
\hline 100 & 12,539 & 12,935 & 12,638 & 12,443 \\
\hline 110 & 8,003 & 7,843 & 7,604 & 7,319 \\
\hline \multirow[t]{2}{*}{120} & 4,102 & 4,256 & 4,202 & 3,961 \\
\hline & \multicolumn{4}{|c|}{$\mathrm{k}=0,5 \%$} \\
\hline Strike & 6 & 13 & 52 & 150 \\
\hline 80 & 27,604 & 27,534 & 27,384 & 27,290 \\
\hline 90 & 19,531 & 19,333 & 18,889 & 18,434 \\
\hline 100 & 12,182 & 12,445 & 11,617 & 10,603 \\
\hline 110 & 7,614 & 7,269 & 6,374 & 4,942 \\
\hline \multirow[t]{2}{*}{120} & 3,754 & 3,726 & 3,077 & 1,870 \\
\hline & \multicolumn{4}{|c|}{$\mathrm{k}=2 \%$} \\
\hline Strike & 6 & 13 & 52 & 150 \\
\hline 80 & 27,327 & 27,276 & $*$ & $*$ \\
\hline 90 & 18,697 & 18,281 & $*$ & $*$ \\
\hline 100 & 10,614 & 10,115 & $*$ & $*$ \\
\hline 110 & 5,845 & 4,311 & $*$ & $*$ \\
\hline 120 & 2,269 & 1,285 & $*$ & $*$ \\
\hline
\end{tabular}

Fuente: elaboración propia.

\subsection{Costos combinados (fijos más proporcionales)}

Tabla 12: Resultados valoración opción europea larga de venta (costos combinados)

Opción europea larga de venta (long put)

\begin{tabular}{|c|c|c|c|c|}
\hline \multirow{2}{*}{} & \multicolumn{4}{|c|}{ Número de nodos (n) } \\
\cline { 2 - 5 } Strike & $\mathbf{6}$ & $\mathbf{1 3}$ & $\mathbf{5 2}$ & $\mathbf{1 5 0}$ \\
\hline 80 & 1,162 & 2,042 & 7,145 & 21,018 \\
\hline 90 & 2,371 & 3,172 & 8,238 & 22,111 \\
\hline 100 & 4,296 & 5,616 & 10,615 & 24,504 \\
\hline 110 & 8,861 & 9,640 & 14,725 & 28,571 \\
\hline
\end{tabular}




\begin{tabular}{|c|c|c|c|c|}
\hline 120 & 14,038 & 15,132 & 20,392 & 34,264 \\
\hline & \multicolumn{4}{|c|}{$\mathrm{k}=0,125 \%$} \\
\hline Strike & 6 & 13 & 52 & 150 \\
\hline 80 & 1,194 & 2,087 & 7,209 & 20,978 \\
\hline 90 & 2,444 & 3,273 & 8,412 & 22,249 \\
\hline 100 & 4,410 & 5,770 & 10,895 & 24,815 \\
\hline 110 & 8,985 & 9,817 & 15,053 & 28,964 \\
\hline \multirow[t]{2}{*}{120} & 14,151 & 15,298 & 20,702 & 34,630 \\
\hline & \multicolumn{4}{|c|}{$\mathrm{k}=0,5 \%$} \\
\hline Strike & 6 & 13 & 52 & 150 \\
\hline 80 & 1,295 & 2,229 & 7,442 & 21,058 \\
\hline 90 & 2,662 & 3,575 & 8,939 & 22,778 \\
\hline 100 & 4,747 & 6,216 & 11,688 & 25,737 \\
\hline 110 & 9,348 & 10,329 & 15,968 & 30,076 \\
\hline \multirow[t]{2}{*}{120} & 14,484 & 15,783 & 21,579 & 35,697 \\
\hline & \multicolumn{4}{|c|}{$\mathrm{k}=2 \%$} \\
\hline Strike & 6 & 13 & 52 & 150 \\
\hline 80 & 1,752 & 2,886 & 8,655 & 22,533 \\
\hline 90 & 3,529 & 4,760 & 10,981 & 25,324 \\
\hline 100 & 6,005 & 7,838 & 14,396 & 29,109 \\
\hline 110 & 10,692 & 12,150 & 18,988 & 33,869 \\
\hline 120 & 15,744 & 17,546 & 24,560 & 39,497 \\
\hline
\end{tabular}

Fuente: elaboración propia.

Tabla 13: Resultados valoración opción europea corta de venta (costos combinados)

Opción europea corta de venta (short put)

\begin{tabular}{|c|c|c|c|c|}
\hline \multirow{2}{*}{} & \multicolumn{4}{|c|}{ Número de nodos (n) } \\
\cline { 2 - 5 } Strike & $\mathbf{6}$ & $\mathbf{1 3}$ & $\mathbf{5 2}$ & $\mathbf{1 5 0}$ \\
\hline 80 & 1,140 & 1,973 & 6,586 & 18,281 \\
\hline 90 & 2,349 & 3,103 & 7,680 & 19,374 \\
\hline 100 & 4,274 & 5,546 & 10,057 & 21,767 \\
\hline
\end{tabular}




\begin{tabular}{|c|c|c|c|c|}
\hline 110 & 8,839 & 9,570 & 14,166 & 25,833 \\
\hline \multirow[t]{2}{*}{120} & 14,016 & 15,062 & 19,834 & 31,527 \\
\hline & \multicolumn{4}{|c|}{$\mathrm{k}=0,125 \%$} \\
\hline Strike & 6 & 13 & 52 & 150 \\
\hline 80 & 1,108 & 1,928 & 6,504 & 18,144 \\
\hline 90 & 2,277 & 3,001 & 7,482 & 19,041 \\
\hline 100 & 4,158 & 5,389 & 9,742 & 21,231 \\
\hline 110 & 8,713 & 9,387 & 13,798 & 25,198 \\
\hline \multirow[t]{2}{*}{120} & 13,902 & 14,891 & 19,488 & 30,931 \\
\hline & \multicolumn{4}{|c|}{$\mathrm{k}=0,5 \%$} \\
\hline Strike & 6 & 13 & 52 & 150 \\
\hline 80 & 1,019 & 1,805 & 6,306 & 17,897 \\
\hline 90 & 2,059 & 2,695 & 6,902 & 18,131 \\
\hline 100 & 3,801 & 4,898 & 8,721 & 19,367 \\
\hline 110 & 8,324 & 8,813 & 12,569 & 22,821 \\
\hline \multirow[t]{2}{*}{120} & 13,554 & 14,361 & 18,363 & 28,840 \\
\hline & \multicolumn{4}{|c|}{$\mathrm{k}=2 \%$} \\
\hline Strike & 6 & 13 & 52 & 150 \\
\hline 80 & 0,764 & 1,548 & $*$ & $*$ \\
\hline 90 & 1,225 & 1,643 & * & $*$ \\
\hline 100 & 2,233 & 2,568 & $*$ & $*$ \\
\hline 110 & 6,555 & 5,856 & $*$ & $*$ \\
\hline 120 & 12,070 & 11,920 & $*$ & $*$ \\
\hline
\end{tabular}

Fuente: elaboración propia.

Tabla 14: Resultados valoración opción americana larga de venta (costos combinados)

Opción americana larga de venta (long put)

\begin{tabular}{|c|c|c|c|c|}
\hline \multirow{2}{*}{} & \multicolumn{4}{|c|}{ Número de nodos (n) } \\
\cline { 2 - 5 } Strike & $\mathbf{6}=\mathbf{0} \%$ \\
\hline 80 & 1,181 & 2,072 & 7,147 & 21,018 \\
\hline 90 & 2,579 & 3,340 & 8,262 & 22,111 \\
\hline
\end{tabular}




\begin{tabular}{|c|c|c|c|c|}
\hline 100 & 5,357 & 6,281 & 10,741 & 24,504 \\
\hline 110 & 11,096 & 11,564 & 15,136 & 28,571 \\
\hline \multirow[t]{2}{*}{120} & 18,610 & 19,548 & 21,547 & 34,264 \\
\hline & \multicolumn{4}{|c|}{$\mathrm{k}=0,125 \%$} \\
\hline Strike & 6 & 13 & 52 & 150 \\
\hline 80 & 1,214 & 2,119 & 7,211 & 20,978 \\
\hline 90 & 2,659 & 3,450 & 8,439 & 22,249 \\
\hline 100 & 5,468 & 6,441 & 11,023 & 24,815 \\
\hline 110 & 11,201 & 11,713 & 15,464 & 28,964 \\
\hline \multirow[t]{2}{*}{120} & 18,692 & 19,629 & 21,818 & 34,630 \\
\hline & \multicolumn{4}{|c|}{$\mathrm{k}=0,5 \%$} \\
\hline Strike & 6 & 13 & 52 & 150 \\
\hline 80 & 1,315 & 2,271 & 7,446 & 21,058 \\
\hline 90 & 2,895 & 3,775 & 8,975 & 22,778 \\
\hline 100 & 5,792 & 6,893 & 11,827 & 25,737 \\
\hline 110 & 11,514 & 12,179 & 16,381 & 30,076 \\
\hline \multirow[t]{2}{*}{120} & 18,939 & 19,879 & 22,610 & 35,697 \\
\hline & \multicolumn{4}{|c|}{$\mathrm{k}=2 \%$} \\
\hline Strike & 6 & 13 & 52 & 150 \\
\hline 80 & 1,793 & 2,967 & 8,667 & 22,533 \\
\hline 90 & 3,815 & 5,040 & 11,050 & 25,324 \\
\hline 100 & 6,976 & 8,598 & 14,576 & 29,109 \\
\hline 110 & 12,663 & 13,828 & 19,421 & 33,869 \\
\hline 120 & 19,912 & 20,958 & 25,454 & 39,497 \\
\hline
\end{tabular}

Fuente: elaboración propia. 
Tabla 15: Resultados valoración opción americana corta de venta (costos combinados)

Opción americana corta de venta (short put)

\begin{tabular}{|c|c|c|c|c|}
\hline \multirow[b]{3}{*}{ Strike } & \multicolumn{4}{|c|}{ Número de nodos (n) } \\
\hline & \multicolumn{4}{|c|}{$k=0 \%$} \\
\hline & 6 & 13 & 52 & 150 \\
\hline 80 & 1,160 & 2,002 & 6,590 & 18,281 \\
\hline 90 & 2,558 & 3,273 & 7,716 & 19,374 \\
\hline 100 & 5,339 & 6,222 & 10,225 & 21,767 \\
\hline 110 & 11,084 & 11,524 & 14,688 & 25,833 \\
\hline \multirow[t]{2}{*}{120} & 18,600 & 19,527 & 21,246 & 31,527 \\
\hline & \multicolumn{4}{|c|}{$\mathrm{k}=0,125 \%$} \\
\hline Strike & 6 & 13 & 52 & 150 \\
\hline 80 & 1,127 & 1,955 & 6,508 & 18,144 \\
\hline 90 & 2,479 & 3,162 & 7,514 & 19,041 \\
\hline 100 & 5,226 & 6,056 & 9,910 & 21,231 \\
\hline 110 & 10,980 & 11,384 & 14,329 & 25,198 \\
\hline \multirow[t]{2}{*}{120} & 18,516 & 19,446 & 20,977 & 30,931 \\
\hline & \multicolumn{4}{|c|}{$\mathrm{k}=0,5 \%$} \\
\hline Strike & 6 & 13 & 52 & 150 \\
\hline 80 & 1,035 & 1,824 & 6,310 & 17,897 \\
\hline 90 & 2,238 & 2,831 & 6,922 & 18,131 \\
\hline 100 & 4,877 & 5,533 & 8,885 & 19,367 \\
\hline 110 & 10,658 & 10,997 & 13,138 & 22,829 \\
\hline \multirow[t]{2}{*}{120} & 18,297 & 19,223 & 20,244 & 28,840 \\
\hline & \multicolumn{4}{|c|}{$\mathrm{k}=2 \%$} \\
\hline Strike & 6 & 13 & 52 & 150 \\
\hline 80 & 0,768 & 1,548 & $*$ & $*$ \\
\hline 90 & 1,294 & 1,659 & $*$ & $*$ \\
\hline 100 & 3,361 & 2,846 & $*$ & $*$ \\
\hline 110 & 9,364 & 9,377 & $*$ & $*$ \\
\hline 120 & 18,232 & 19,248 & $*$ & $*$ \\
\hline
\end{tabular}

Fuente: elaboración propia. 
Tabla 16: Resultados valoración opción europea larga de compra (costos combinados)

Opción europea larga de compra (long call)

\begin{tabular}{|c|c|c|c|c|}
\hline \multirow[b]{3}{*}{ Strike } & \multicolumn{4}{|c|}{ Número de nodos (n) } \\
\hline & \multicolumn{4}{|c|}{$k=0 \%$} \\
\hline & 6 & 13 & 52 & 150 \\
\hline 80 & 28,434 & 29,315 & 34,418 & 48,291 \\
\hline 90 & 20,553 & 21,354 & 26,420 & 40,293 \\
\hline 100 & 13,387 & 14,707 & 19,706 & 33,595 \\
\hline 110 & 8,861 & 9,640 & 14,725 & 28,571 \\
\hline \multirow[t]{2}{*}{120} & 4,947 & 6,041 & 11,301 & 25,173 \\
\hline & \multicolumn{4}{|c|}{$\mathrm{k}=0,125 \%$} \\
\hline Strike & 6 & 13 & 52 & 150 \\
\hline 80 & 28,467 & 29,360 & 34,482 & 48,251 \\
\hline 90 & 20,626 & 21,455 & 26,594 & 40,431 \\
\hline 100 & 13,501 & 14,860 & 19,985 & 33,906 \\
\hline 110 & 8,985 & 9,817 & 15,053 & 28,964 \\
\hline \multirow[t]{2}{*}{120} & 5,060 & 6,207 & 11,611 & 25,539 \\
\hline & \multicolumn{4}{|c|}{$\mathrm{k}=0,5 \%$} \\
\hline Strike & 6 & 13 & 52 & 150 \\
\hline 80 & 28,567 & 29,502 & 34,715 & 48,331 \\
\hline 90 & 20,844 & 21,757 & 27,121 & 40,960 \\
\hline 100 & 13,838 & 15,307 & 20,779 & 34,828 \\
\hline 110 & 9,348 & 10,329 & 15,968 & 30,076 \\
\hline \multirow[t]{2}{*}{120} & 5,393 & 6,692 & 12,488 & 26,606 \\
\hline & \multicolumn{4}{|c|}{$\mathrm{k}=2 \%$} \\
\hline Strike & 6 & 13 & 52 & 150 \\
\hline 80 & 29,024 & 30,159 & 35,928 & 49,806 \\
\hline 90 & 21,711 & 22,941 & 29,162 & 43,506 \\
\hline 100 & 15,096 & 16,929 & 23,487 & 38,200 \\
\hline 110 & 10,692 & 12,150 & 18,988 & 33,869 \\
\hline 120 & 6,653 & 8,455 & 15,469 & 30,406 \\
\hline
\end{tabular}

Fuente: elaboración propia. 
Tabla 17: Resultados valoración opción europea corta de compra (costos combinados)

Opción europea corta de compra (short call)

\begin{tabular}{|c|c|c|c|c|}
\hline \multirow[b]{3}{*}{ Strike } & \multicolumn{4}{|c|}{ Número de nodos (n) } \\
\hline & \multicolumn{4}{|c|}{$k=0 \%$} \\
\hline & 6 & 13 & 52 & 150 \\
\hline 80 & 28,413 & 29,246 & 33,859 & 45,554 \\
\hline 90 & 20,531 & 21,285 & 25,861 & 37,555 \\
\hline 100 & 13,365 & 14,637 & 19,147 & 30,858 \\
\hline 110 & 8,839 & 9,570 & 14,166 & 25,833 \\
\hline \multirow[t]{2}{*}{120} & 4,925 & 5,971 & 10,743 & 22,436 \\
\hline & \multicolumn{4}{|c|}{$\mathrm{k}=0,125 \%$} \\
\hline Strike & 6 & 13 & 52 & 150 \\
\hline 80 & 28,381 & 29,201 & 33,777 & 45,417 \\
\hline 90 & 20,458 & 21,182 & 25,664 & 37,223 \\
\hline 100 & 13,249 & 14,479 & 18,833 & 30,322 \\
\hline 110 & 8,713 & 9,387 & 13,798 & 25,198 \\
\hline \multirow[t]{2}{*}{120} & 4,811 & 5,800 & 10,397 & 21,840 \\
\hline & \multicolumn{4}{|c|}{$\mathrm{k}=0,5 \%$} \\
\hline Strike & 6 & 13 & 52 & 150 \\
\hline 80 & 28,291 & 29,078 & 33,579 & 45,169 \\
\hline 90 & 20,241 & 20,877 & 25,084 & 36,313 \\
\hline 100 & 12,892 & 13,989 & 17,812 & 28,457 \\
\hline 110 & 8,324 & 8,813 & 12,569 & 22,821 \\
\hline \multirow[t]{2}{*}{120} & 4,464 & 5,270 & 9,272 & 19,750 \\
\hline & \multicolumn{4}{|c|}{$\mathrm{k}=2 \%$} \\
\hline Strike & 6 & 13 & 52 & 150 \\
\hline 80 & 28,037 & 28,821 & $*$ & $*$ \\
\hline 90 & 19,407 & 19,825 & $*$ & $*$ \\
\hline 100 & 11,324 & 11,659 & $*$ & $*$ \\
\hline 110 & 6,555 & 5,856 & $*$ & $*$ \\
\hline 120 & 2,979 & 2,829 & $*$ & $*$ \\
\hline
\end{tabular}

Fuente: elaboración propia. 
Tabla 18: Resultados valoración opción americana larga de compra (costos combinados)

Opción americana larga de compra (long call)

\begin{tabular}{|c|c|c|c|c|}
\hline \multirow[b]{3}{*}{ Strike } & \multicolumn{4}{|c|}{ Número de nodos (n) } \\
\hline & \multicolumn{4}{|c|}{$k=0 \%$} \\
\hline & 6 & 13 & 52 & 150 \\
\hline 80 & 28,434 & 29,315 & 34,418 & 48,291 \\
\hline 90 & 20,553 & 21,354 & 26,420 & 40,293 \\
\hline 100 & 13,387 & 14,707 & 19,706 & 33,595 \\
\hline 110 & 8,861 & 9,640 & 14,725 & 28,571 \\
\hline \multirow[t]{2}{*}{120} & 4,947 & 6,041 & 11,301 & 25,173 \\
\hline & \multicolumn{4}{|c|}{$\mathrm{k}=0,125 \%$} \\
\hline Strike & 6 & 13 & 52 & 150 \\
\hline 80 & 28,467 & 29,360 & 34,482 & 48,251 \\
\hline 90 & 20,626 & 21,455 & 26,594 & 40,431 \\
\hline 100 & 13,501 & 14,860 & 19,985 & 33,906 \\
\hline 110 & 8,985 & 9,817 & 15,053 & 28,964 \\
\hline \multirow[t]{2}{*}{120} & 5,060 & 6,207 & 11,611 & 25,539 \\
\hline & \multicolumn{4}{|c|}{$\mathrm{k}=0,5 \%$} \\
\hline Strike & 6 & 13 & 52 & 150 \\
\hline 80 & 28,567 & 29,502 & 34,715 & 48,331 \\
\hline 90 & 20,844 & 21,757 & 27,121 & 40,960 \\
\hline 100 & 13,838 & 15,307 & 20,779 & 34,828 \\
\hline 110 & 9,348 & 10,329 & 15,968 & 30,076 \\
\hline \multirow[t]{2}{*}{120} & 5,393 & 6,692 & 12,488 & 26,606 \\
\hline & \multicolumn{4}{|c|}{$\mathrm{k}=2 \%$} \\
\hline Strike & 6 & 13 & 52 & 150 \\
\hline 80 & 29,024 & 30,159 & 35,928 & 49,806 \\
\hline 90 & 21,711 & 22,941 & 29,162 & 43,506 \\
\hline 100 & 15,096 & 16,929 & 23,487 & 38,200 \\
\hline 110 & 10,692 & 12,150 & 18,988 & 33,869 \\
\hline 120 & 6,653 & 8,455 & 15,469 & 30,406 \\
\hline
\end{tabular}

Fuente: elaboración propia. 
Tabla 19: Resultados valoración opción americana corta de compra (costos combinados)

Opción americana corta de compra (short call)

\begin{tabular}{|c|c|c|c|c|}
\hline \multirow[b]{3}{*}{ Strike } & \multicolumn{4}{|c|}{ Número de nodos (n) } \\
\hline & \multicolumn{4}{|c|}{$\mathrm{k}=\mathbf{0} \%$} \\
\hline & 6 & 13 & 52 & 150 \\
\hline 80 & 28,413 & 29,246 & 33,859 & 45,554 \\
\hline 90 & 20,531 & 21,285 & 25,861 & 37,555 \\
\hline 100 & 13,365 & 14,637 & 19,147 & 30,858 \\
\hline 110 & 8,839 & 9,570 & 14,166 & 25,833 \\
\hline \multirow[t]{2}{*}{120} & 4,925 & 5,971 & 10,743 & 22,436 \\
\hline & \multicolumn{4}{|c|}{$\mathrm{k}=0,125 \%$} \\
\hline Strike & 6 & 13 & 52 & 150 \\
\hline 80 & 28,381 & 29,201 & 33,777 & 45,417 \\
\hline 90 & 20,458 & 21,182 & 25,664 & 37,223 \\
\hline 100 & 13,249 & 14,479 & 18,833 & 30,322 \\
\hline 110 & 8,713 & 9,387 & 13,798 & 25,198 \\
\hline \multirow[t]{2}{*}{120} & 4,811 & 5,800 & 10,397 & 21,840 \\
\hline & \multicolumn{4}{|c|}{$\mathrm{k}=0,5 \%$} \\
\hline Strike & 6 & 13 & 52 & 150 \\
\hline 80 & 28,291 & 29,078 & 33,579 & 45,169 \\
\hline 90 & 20,241 & 20,877 & 25,084 & 36,313 \\
\hline 100 & 12,892 & 13,989 & 17,812 & 28,461 \\
\hline 110 & 8,324 & 8,813 & 12,569 & 22,821 \\
\hline \multirow[t]{2}{*}{120} & 4,464 & 5,270 & 9,272 & 19,750 \\
\hline & \multicolumn{4}{|c|}{$\mathrm{k}=2 \%$} \\
\hline Strike & 6 & 13 & 52 & 150 \\
\hline 80 & 28,037 & 28,821 & $*$ & $*$ \\
\hline 90 & 19,407 & 19,825 & $*$ & $*$ \\
\hline 100 & 11,324 & 11,659 & $*$ & $*$ \\
\hline 110 & 6,555 & 5,856 & * & $*$ \\
\hline 120 & 2,979 & 2,829 & $*$ & $*$ \\
\hline
\end{tabular}

Fuente: elaboración propia. 


\subsection{Análisis de resultados}

Realizadas las simulaciones de los modelos en Excel-Visual Basic, se lleva a cabo el siguiente análisis:

Opciones de compra (call). Al correr el modelo Cox, Ross, Rubinstein sin fricciones de esta naturaleza, el diferencial de las primas en las posiciones largas y cortas de estas opciones es nulo, sin embargo, una vez se incluye esta fricción se observa cómo los costos de transacción causados en cada uno de los ajustes de posiciones generan un diferencial de primas que aumenta conforme se incrementan los costos y los periodos de revisión (figura 1). Es decir, con dichos incrementos el tenedor de la opción paga cada vez una mayor prima al intermediario, mientras su contraparte percibe una cantidad menor.

Además, realizando una comparación entre las primas de las opciones de compra americanas y europeas se observa que son iguales en todos los casos, es decir, no se realiza un ejercicio anticipado de la opción. Consecuentemente, podemos inferir que, en presencia de costos de transacción proporcionales y fijos, el diferencial de primas entre posiciones será el mismo, lo que hace que para el intermediario sea indiferente si el comprador de la opción prefiere tomar una $\mathrm{u}$ otra.

Figura 1: Diferencial de precios opciones call

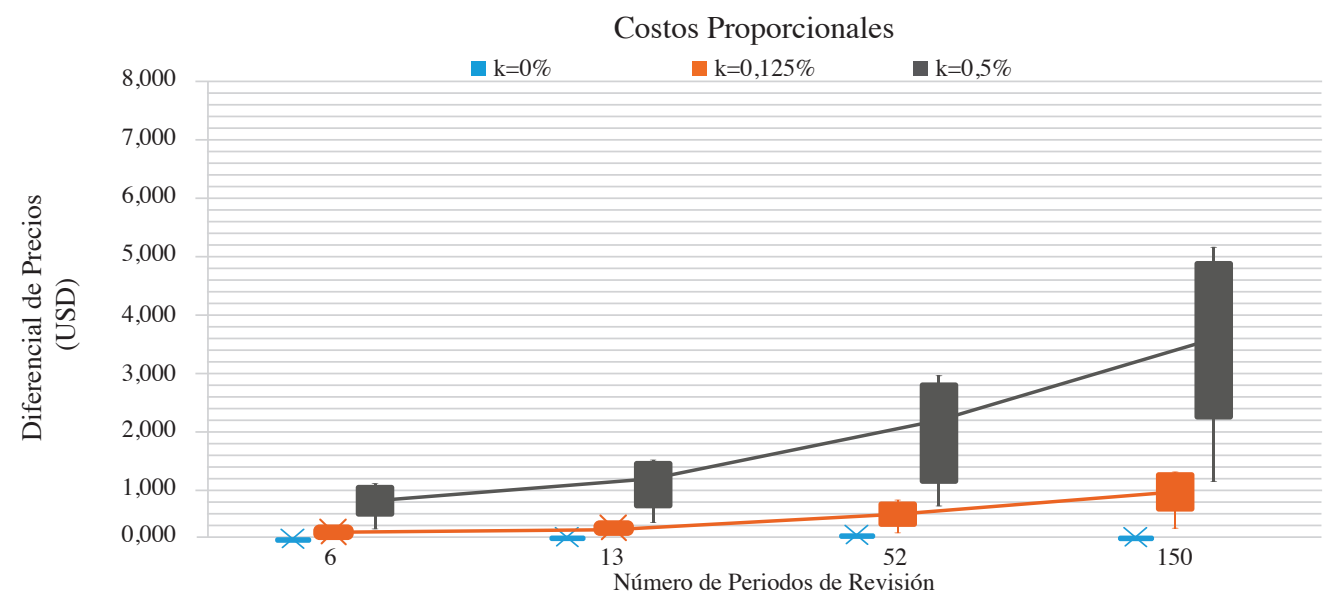




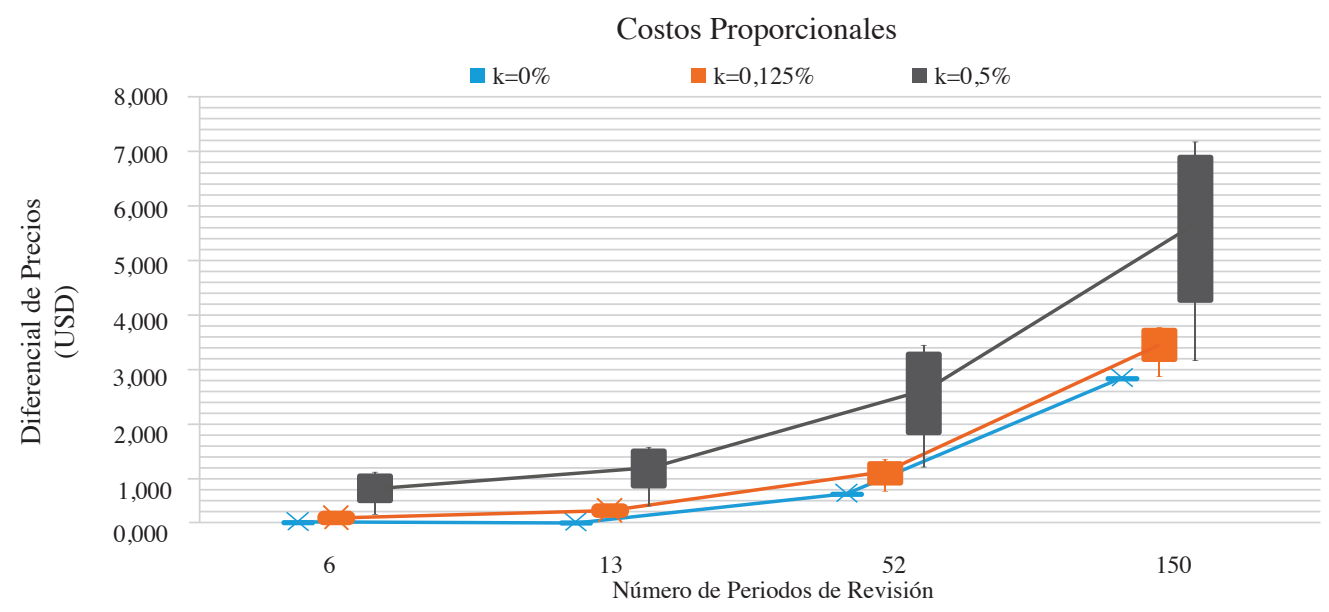

Fuente: elaboración propia.

Por otra parte, si bien se observa que la inclusión de costos fijos y proporcionales tiene un impacto similar sobre el precio de las opciones, es posible notar cómo el diferencial promedio de las primas en las posiciones largas y cortas aumenta sustancialmente una vez se incluyen los costos fijos, principalmente cuando se incrementan los periodos de revisión. De esta manera es posible inferir que un número elevado de rebalanceos hará más ineficiente la posición tanto del tenedor como del vendedor de la opción, lo que beneficia de manera exclusiva al intermediario.

Opciones de venta (put): al igual que en las opciones de compra, cuando se incorporan los costos de transacción, el denominado diferencial de precios entre las posiciones largas y cortas deja de ser nulo, y pasa a incrementarse conforme se aumenta el porcentaje de costos y los periodos de revisión (figura 1). Sin embargo, en las opciones de venta, el ejercicio anticipado de las opciones americanas genera que el diferencial promedio de precios sea mayor que en las opciones europeas, lo cual se traduce en un mayor beneficio para el intermediario.

Asimismo, al igual que en las opciones de compra, el impacto generado por los costos fijos sobre el diferencial de precios es mayor al de los costos proporcionales, lo cual se intensifica paulatinamente conforme se incrementa el número de nodos de revisión (figura 2). Esto hará que el beneficio del intermediario aumente y, consecuentemente, que las posiciones del tenedor y el vendedor del instrumento financiero sean menos eficientes. 
Figura 2: Diferencial de precios opciones put

Costos Proporcionales
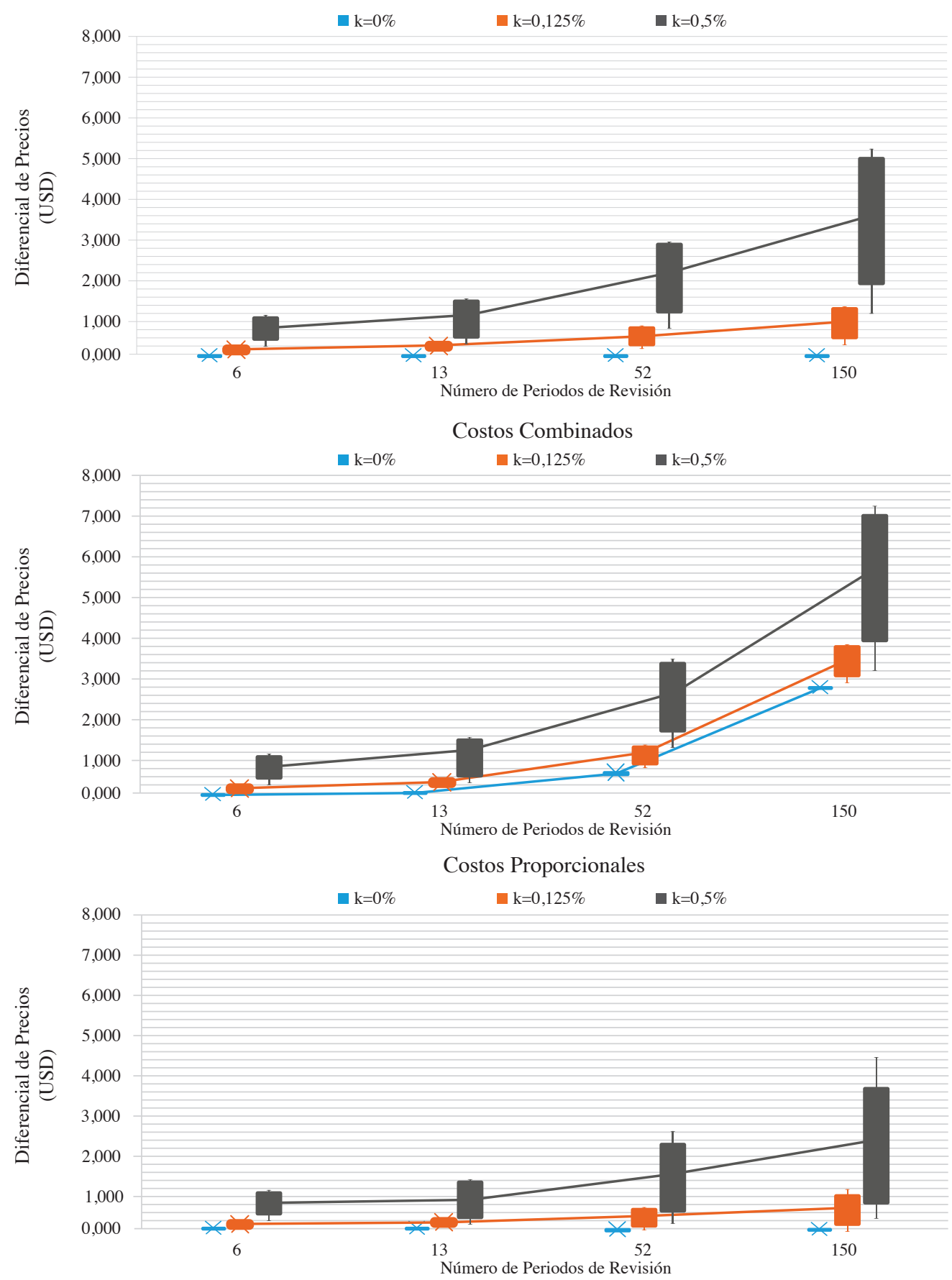

ODEON, ISSN: 1794-1113, E-ISSN: 2346-2140, N 15, julio-diciembre de 2018, pp. 7-52 


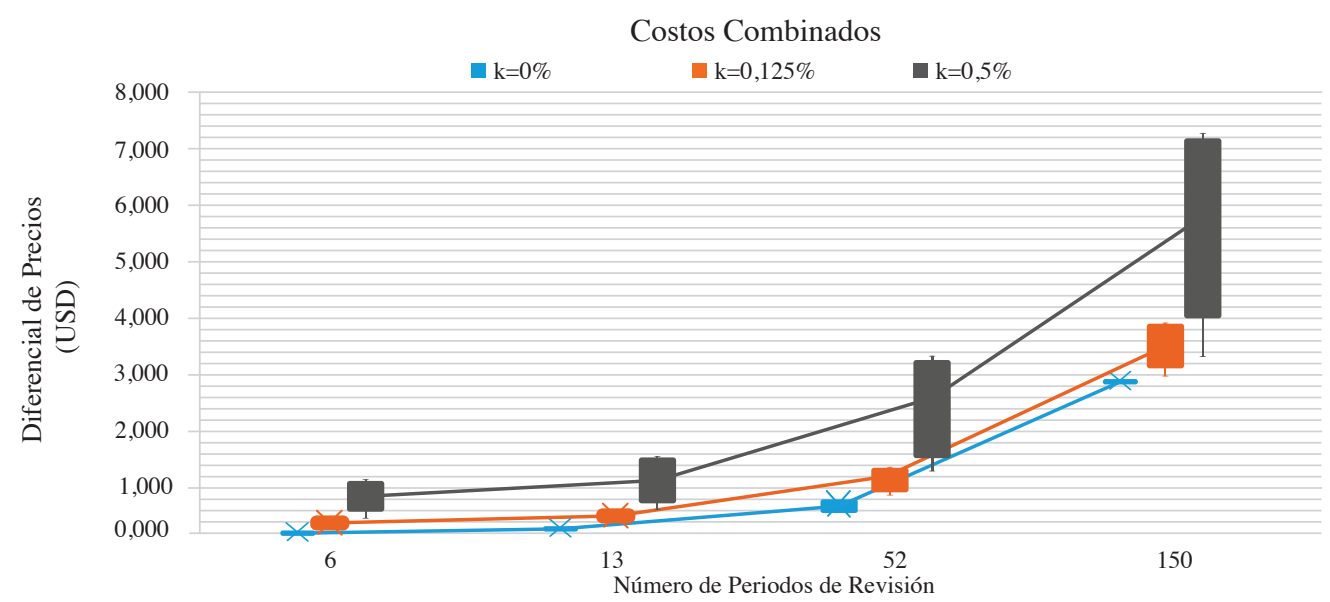

Fuente: elaboración propia.

Por otra parte, a diferencia de las opciones call, la prima de las opciones americanas en la mayoría de los casos será superior a la de las opciones europeas; solo cuando el número de periodos de revisión se aumenta de forma significativa, hasta 150 en el caso de esta simulación, las primas de europeas y americanas tienden a igualarse (figura 3) para todos los strikes y con todos los costos de transacción.

Figura 3: Diferencial de precios americanas vs. europeas, opciones put

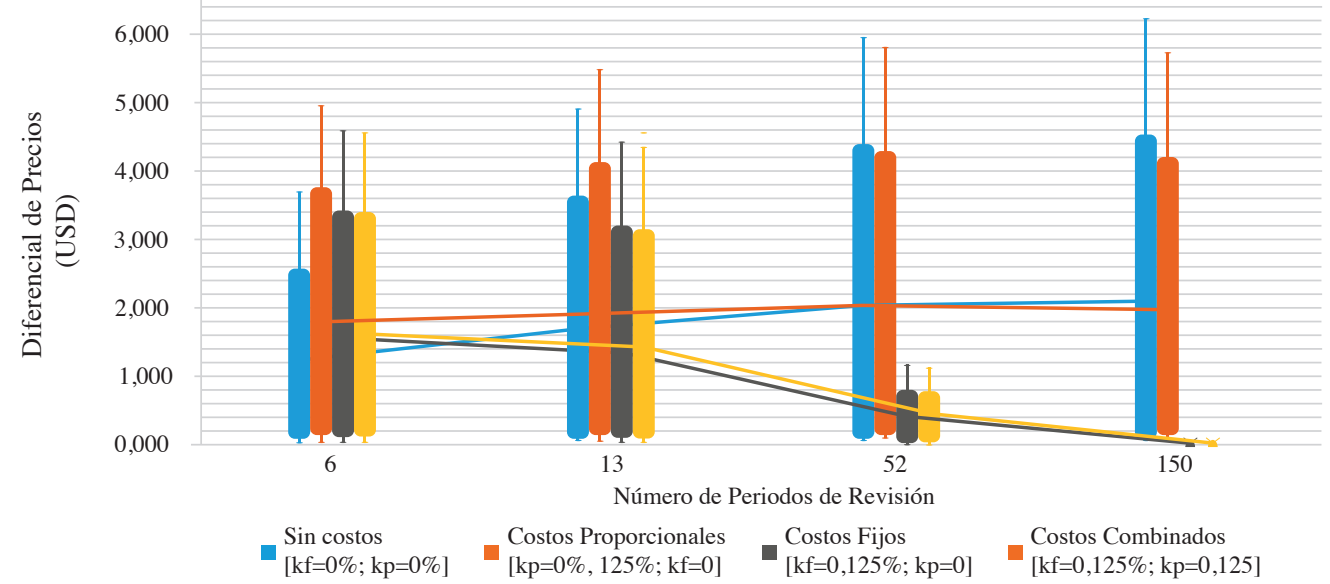

Fuente: elaboración propia. 
Además, cabe destacar que en presencia de costos de transacción proporcionales y combinados el valor de las opciones call disminuye a strikes más altos, sucediendo lo contrario en las opciones put (figura 4). Además, es posible observar cómo la inclusión de costos fijos genera una mayor dispersión en los datos a medida que aumentan los periodos de revisión.

Finalmente, a partir de los resultados obtenidos desde la simulación del modelo CRR, se observó que el ratio de cobertura delta $(\Delta)$ en la valoración de opciones financieras sin costos de transacción indica el ajuste que debe realizar el inversionista en su posición de activo subyacente durante el rebalanceo del portafolio de réplica. Mientras, el ratio de cambio Gamma $(\Gamma)$ muestra la sensibilidad del delta ante cambios en el precio del activo riesgoso. Una vez se incluyen en el modelo los costos de transacción, se observa que los resultados procedentes de la simulación del delta no son congruentes con los datos obtenidos durante el ajuste de posiciones. A pesar de ser una ligera diferencia, se puede concluir que las expresiones para el cálculo de las griegas (4) y (5) no consideran la presencia de costos de transacción, ya sea proporcionales o fijos.

Figura 4: Comportamientos de primas con costos proporcionales y combinados

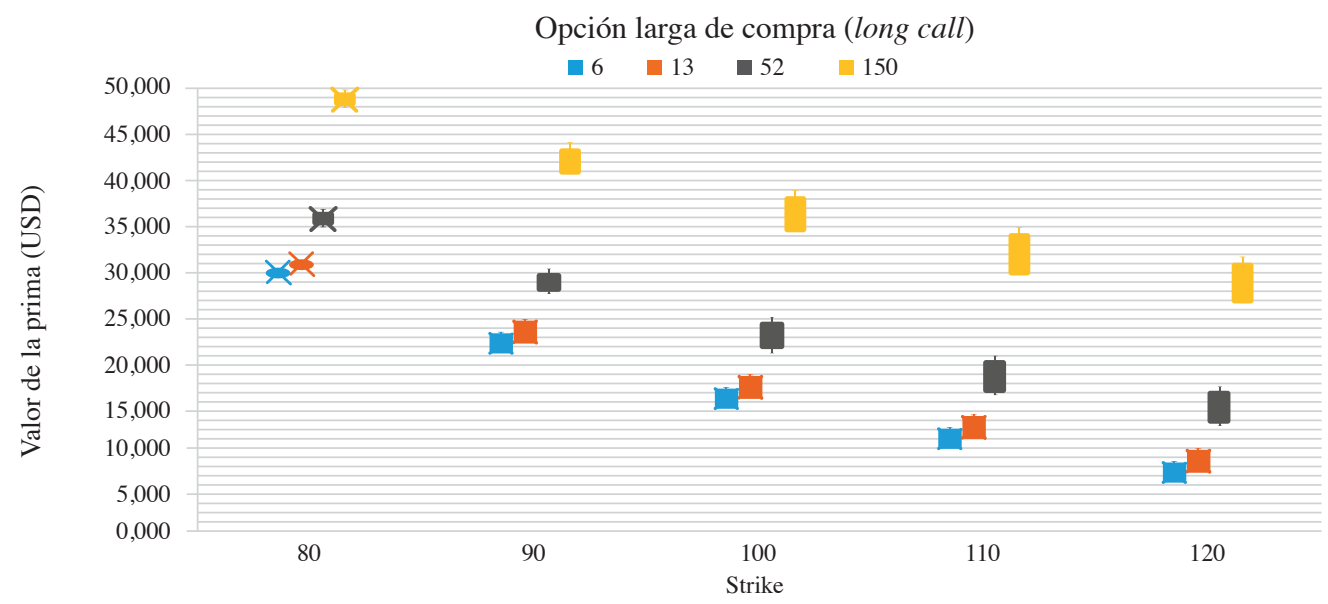




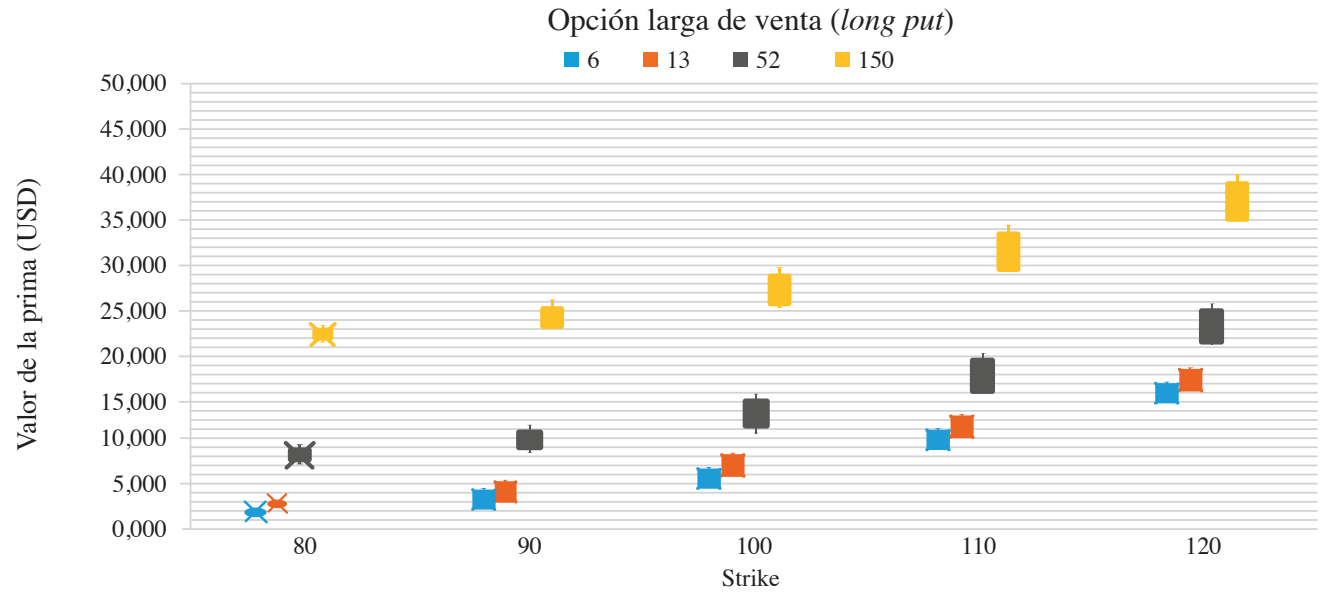

Opción larga de compra (long call)

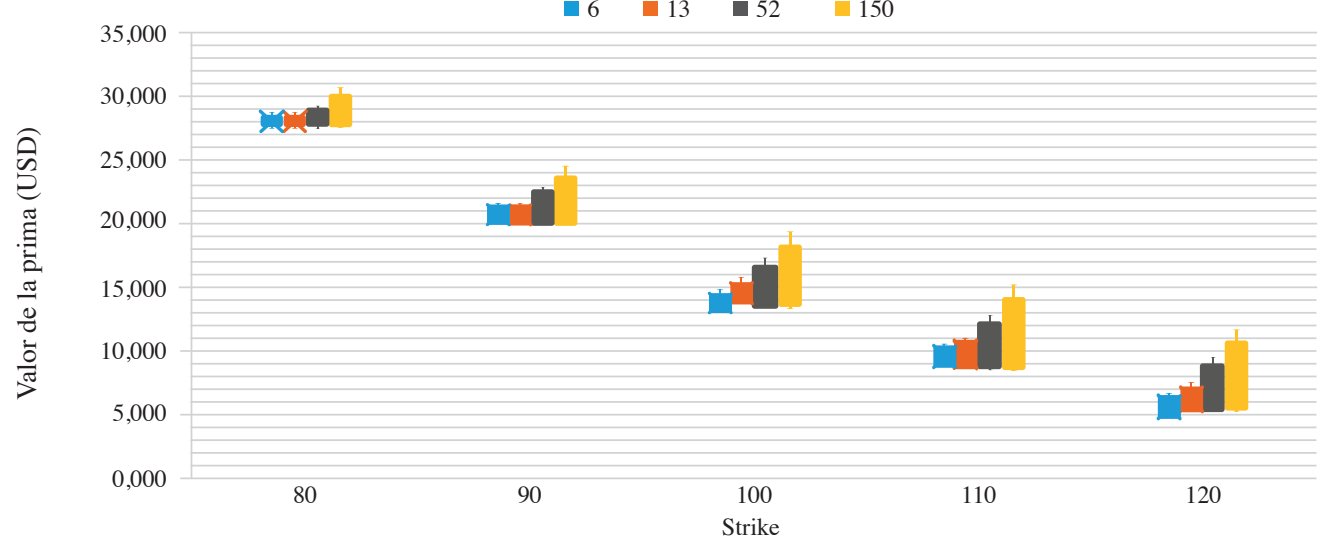

Opción larga de venta (long put)

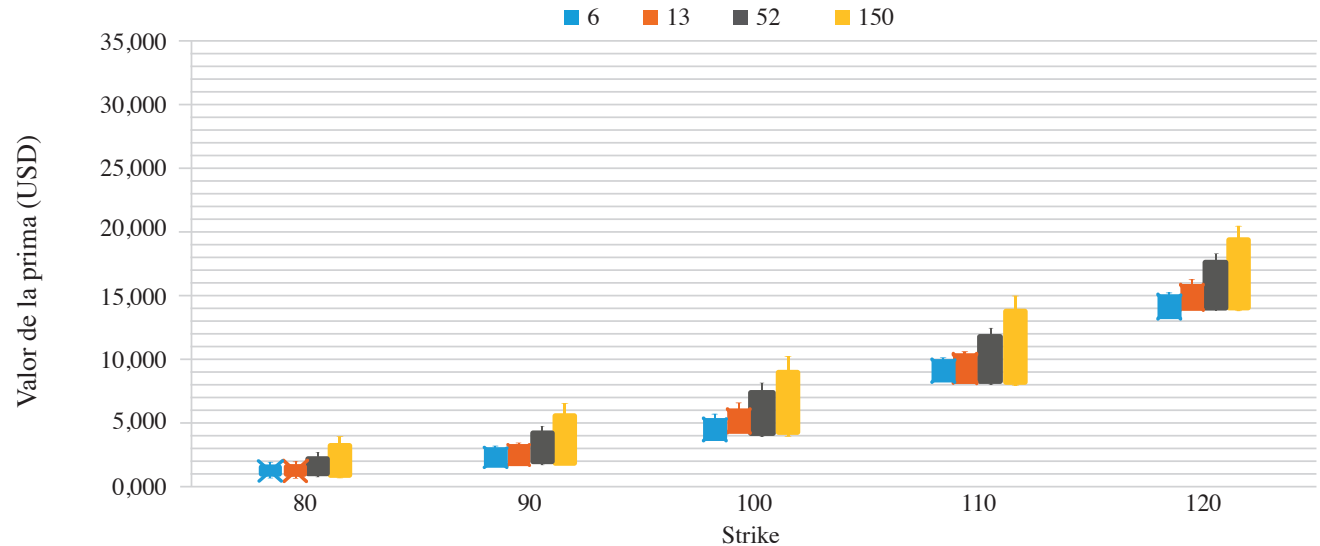

Fuente: elaboración propia.

ODEON, ISSN: 1794-1113, E-ISSN: 2346-2140, N 15, julio-diciembre de 2018, pp. 7-52 


\section{Conclusiones}

En este documento se presenta e implementa una extensión del modelo Cox, Ross y Rubinstein, donde se incluyen dos tipos de costos de transacción como fricciones del mercado durante el ajuste de posiciones en cada periodo. Se determina entonces el impacto en la valoración de opciones europeas y americanas call y put desde las perspectivas tanto del vendedor y el comprador del instrumento financiero como del intermediario (creador de mercado o market maker).

El modelo CRR sin costos de transacción refleja una diferencia nula entre las primas de las posiciones cortas y largas en las opciones financieras evaluadas, es decir, la prima pagada por el tenedor de la opción es la misma percibida por el vendedor en todos los casos. Esto debido a que no existe un costo por la intermediación en el rebalanceo de posiciones durante la construcción del portafolio de réplica, ya sea porque se asume una transacción directa o, lo que es menos probable, que el intermediario decida no cobrar por el riesgo asumido. Cualquiera de las dos supone una restricción muy fuerte en la realidad, que desconoce el posible impacto del número de periodos de revisión sobre el valor de la prima.

Una vez se incluyen en el modelo Cox, Ross, Rubinstein los costos de transacción proporcionales (al monto comercializado), se evidencia cómo, desde la perspectiva del tenedor de la opción, se incrementa el valor que debe pagar por las primas y, paralelamente, cómo se disminuye el valor percibido por estas desde la perspectiva del vendedor del mismo instrumento financiero. Esto genera un diferencial de precios a favor del creador del mercado, quien hace las veces de intermediario en las transacciones necesarias para el ajuste de posiciones. Si bien frente al modelo original esto genera posiciones de compra y venta menos eficientes, hace un mayor acercamiento a la realidad de los mercados y permite dimensionar el impacto generado por la presencia del intermediario. Ahora, la presencia de costos de transacción permite observar cómo un mayor número de rebalanceos, si bien garantiza un mejor ajuste, incrementa el diferencial de precios entre las posiciones de compra y venta. Es posible concluir que, con cero costos de transacción, se dejan de percibir las variaciones en las primas generadas por un cambio en el número de periodos de revisión y, en general, la presencia del creador de mercado.

A diferencia de los proporcionales, la inclusión de costos fijos en el modelo CRR no depende del monto comercializado; por el contrario, su causación está ligada a la necesidad permanente de asegurar la liquidez del activo subyacente. Esto hace que se presente una mayor fricción, que se ve representada en un 
aumento del diferencial promedio de precios en las posiciones de compra y venta de la opción financiera, es decir, mayores costos absorbidos por el creador del mercado. La sensibilidad de las primas a los costos fijos es tal, que un número elevado de periodos de revisión termina generando posiciones totalmente ineficientes, es decir, en las que cubrir el riesgo de iliquidez no justifica el valor de la opción. Este tipo de costos evidencia en mayor medida la fuerza que tiene suponer un mercado libre de fricciones, ya que en la realidad la liquidez del activo no se encuentra asegurada sin un intermediario.

En resumen, la presencia de costos de transacción por sí misma incrementa el diferencial de los precios de compra y venta de las opciones financieras; sin embargo, esto se intensifica cuando en la estrategia de cobertura dinámica se plantea un mayor número de periodos de revisión, lo cual se hace evidente al netear las posiciones del creador de mercado. Teniendo en cuenta que el modelo CRR originalmente no tiene en cuenta esta fricción, podemos decir que obvia el impacto que tiene la iliquidez del activo subyacente y la estrategia de cobertura dinámica para garantizar el mejor ajuste.

Finalmente, como posibles extensiones del presente trabajo se plantean dos alternativas: i) la inclusión de los costos de transacción en la metodología de opciones reales para la evaluación de proyectos de inversión, con el objetivo de concluir acerca de la sensibilidad del valor del proyecto ante fricciones del mercado que puedan ser modeladas como costos de transacción fijos o proporcionales; y ii) ajustar la fórmula de cálculo del ratio de cobertura delta $(\Delta)$ y el ratio de cambio Gamma $(\Gamma)$ para opciones financieras con costos de transacción tanto proporcionales al monto de la transacción como fijos en tiempo discreto.

\section{Referencias}

Berk, J. y Demarzo, P. (2008). Finanzas Corporativas (pp. 656-686). México: Pearson Educación.

Boyle, P. P. y Vorst, T. (1992). Option replication in discrete time with transaction costs. The Journal of Finance, XLVII, 271-293.

Moreno T., J. F. (2015). Modelos de valoración de dos estados. Cuadernos del CIPE, 24, 5-15. Bogotá: Universidad Externado de Colombia. 
Rogers, L. C. G. y Talay, D. (1999). Numerical Methods in Finance. Cambridge: Cambridge University Press.

Stoll, H. (1989). Inferring the components of the bid-ask spread: Theory and empirical tests. The Journal of Finance, 44(1), 115-134.

Tichý, T. (2005). Binomial model and transaction costs. International Conference Finance for Business and Financial Institutions, vŠB (401-416). Technical University of Ostrava, Faculty of Economics, Department of Finance.

Wallner, C. y Wystup, U. (2004). Efficient computation of option price sensitivities for options of American style. CPQF Working Paper Series, 1, 6-7. Frankfurt, M.: HfB, Business School of Finance and Management. 